\title{
New species and records of Salticidae (Aranei) from Turkmenistan and neighbouring countries
}

\section{Новые виды и находки Salticidae (Aranei) из Туркменистана и прияегающих стран}

\author{
Dmitri V. Logunov ${ }^{1 *}$, Alexander V. Ponomarev ${ }^{2}$ \\ Амитрий В. Аогунов ${ }^{1 *}$, Александр В. Пономарёв ${ }^{2}$
}

\footnotetext{
${ }^{1}$ The Manchester Museum, the University of Manchester, Oxford Road, Manchester M13 9PL, UK.

Email: dmitri.v.logunov@manchester.ac.uk

${ }^{2}$ Southern Scientific Centre of the Russian Academy of Sciences, Chekhov street 41, Rostov-on-Don, 344006, Russia. Email: ponomarev1952@mail.ru

* The corresponding author.

${ }^{1}$ Манчестерский Музей, Университет Манчестера, Оксфорд роуд, Манчестер M13 9PL, Великобритания.

2 Южный научный центр РАН, пр. Чехова, 41, Ростов-на-Дону 344006, Россия.

* Автор для переписки.
}

KEY WORDS: Araneae, Central Asia, description, diagnosis, fauna, Iran, jumping spiders, Kyrgyzstan, Pakistan, Tajikistan, Uzbekistan.

КЛЮЧЕВЫЕ СЛОВА: Araneae, Центральная Азия, описание, диагноз, фауна, Иран, пауки-скакунчики, Кыргызстан, Пакистан, Таджикистан, Узбекистан.

ABSTRACT. New faunistic records are provided for 33 species of Salticidae from Turkmenistan, with additional records for 12 species from such neighbouring central Asian countries as Iran, Kyrgyzstan, Pakistan, Tajikistan and Uzbekistan. A new species of the jumping spiders - Salticus karakumensis sp.n. $\left(\mathrm{O}^{7}\right)$ from Repetek (Turkmenistan) — is diagnosed, illustrated and described. Four species are recorded from Turkmenistan for the first time: Evarcha nenilini Rakov, 1997, Heliophanus forcipifer Kulczyński, 1895, Marpissa nivoyi (Lucas, 1846), and Pellenes allegrii Caporiacco, 1935. One species - Langona tartarica (Charitonov, 1946) - is recorded from Pakistan for the first time. Six species are illustrated: Afraflacilla braunsi (Peckham et Peckham, 1903), Evarcha nenili$n i$ Rakov, 1997, Langona pallidula Logunov et Rakov, 1998, L. tartarica, Pellenes sytchevskayae Logunov, Marusik et Rakov, 1999, and Pseudomogrus pseudovalidus (Logunov et Marusik, 2003).

How to cite this article: Logunov D.V., Ponomarev A.V. 2020. New species and records of Salticidae (Aranei) from Turkmenistan and neighbouring countries // Arthropoda Selecta. Vol.29. No.1. P.67-81. doi: 10.15298/arthsel. 29.1.05

РЕЗЮМЕ. Приводятся новые фаунистические находки для 33 видов Salticidae из Туркменистана, а для 12 из них еще и дополнительные находки из прилегающих стран Центральной Азии, таких как Иран, Кыргызстан, Пакистан, Таджикистан и Узбекистан. Описан, проиллюстрирован и диагностирован новый вид пауков-скакунчиков Salticus karakumensis sp.n. ( $\left.\sigma^{7}\right)$ из Репетека (Туркменистан). Че- тыре вида указаны впервые для Туркменистана: Evarcha nenilini Rakov, 1997, Heliophanus forcipifer Kulczyński, 1895, Marpissa nivoyi (Lucas, 1846) и Pellenes allegrii Caporiacco, 1935. Один вид - Langona tartarica (Charitonov, 1946) - указан впервые для Пакистана. Шесть видов проиллюстрированы: Afraflacilla braunsi (Peckham et Peckham, 1903), Evarcha nenilini Rakov, 1997, Langona pallidula Logunov et Rakov, 1998, L. tartarica, Pellenes sytchevskayae Logunov, Marusik et Rakov, 1999 и Pseudomogrus pseudovalidus (Logunov et Marusik, 2003).

\section{Introduction}

The spider fauna of Turkmenistan is relatively wellknown, primarily due to taxonomic-faunistic studies of Soviet/Russian scientists in 1930s-2000s. Mikhailov \& Fet [1994] provided a historic account on arachnological studies in Turkmenistan for the period of 1930s1980 s and also published its first spider catalogue. According to these authors, the Salticidae represented the most diverse spider family in Turkmenistan, accounting for $62(19 \%)$ of 334 species recorded from there by 1994 . Later, many papers specially devoted to Salticidae of Turkmenistan and/or Middle Asia were published [Wesołowska, 1996; Logunov, 1996; Rakov, Logunov, 1997a,b; Logunov, Rakov, 1998; Logunov et al., 1999; Logunov, Marusik, 1999, 2000a, 2003; etc.]. As a result, the salticid fauna of Turkmenistan was thoroughly revised, reached 94 recorded/described species and now accounts for $24 \%$ of the total regional spider diversity (394 species) [Mikhailov, 


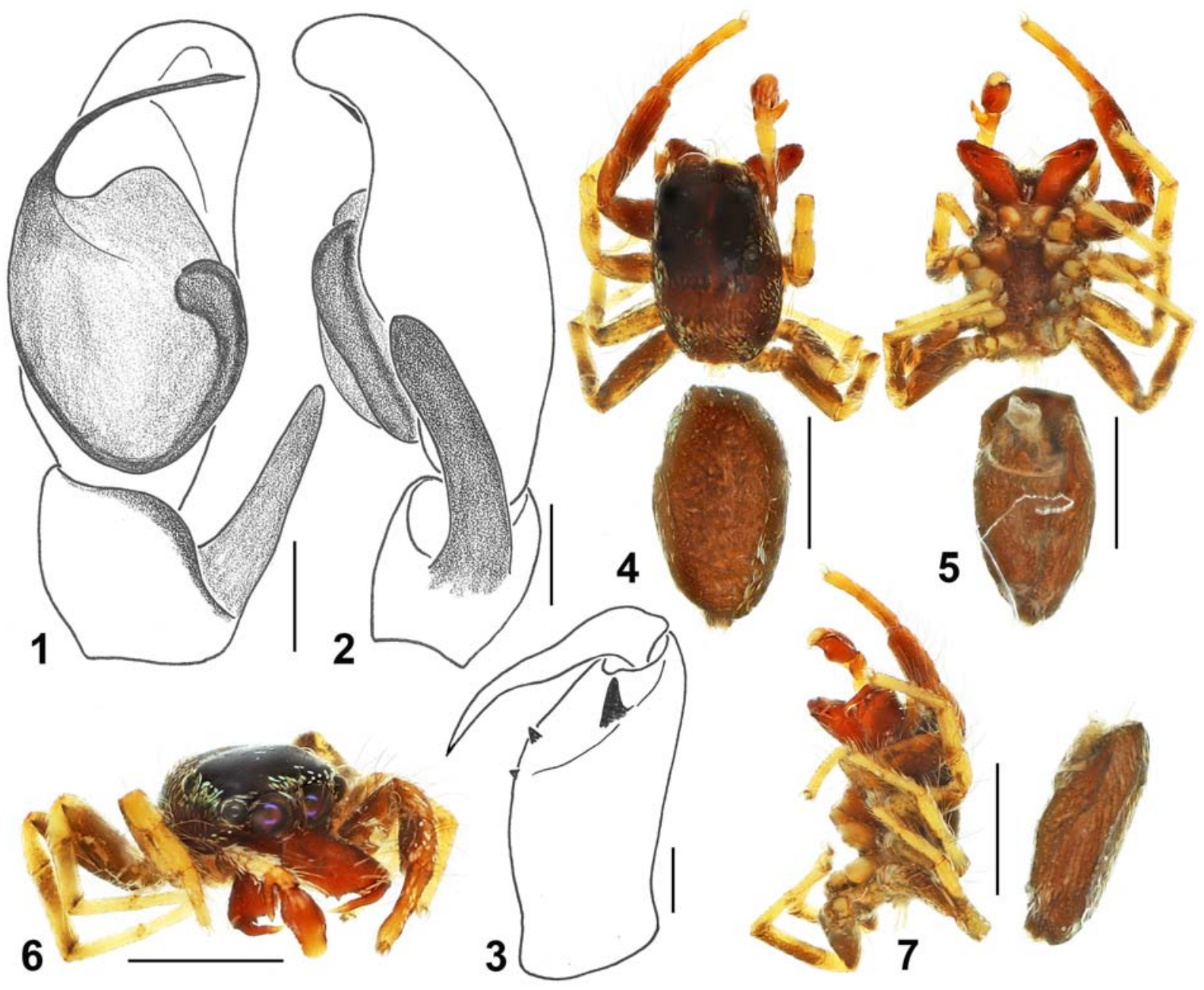

Figs 1-7. Male palp $(1,2)$ and somatic characters (3-7) of the holotype of Salticus karakumensis sp.n.: 1 - ventral view; 2 retrolateral view; 3 - left chelicerae, ventral view; 4 - general appearance, dorsal view; 5 - ditto, ventral view; 6 - carapace, frontal view; 7 - carapace and abdomen, lateral view. Scale bars: (1-3) $0.1 \mathrm{~mm},(4-7) 1 \mathrm{~mm}$.

Рис. 1-7. Пальпа самца $(1,2)$ и соматическеи признаки (3-7) голотипа Salticus karakumensis sp.n.: 1 - вид снизу; 2 - вид сзади-сбоку; 3 - левая пальпа, вид снизу; 4 - общий вид, вид сверху; 5 - тоже, вид снизу; 6 - головогрудь, вид спереди; 7 головогрудь и брюшко, вид сбоку. Масштаб: (1-3) 0,1 мм, (4-7) 1 мм.

2016]. Since 2003 till now, there have been no publications on the Turkmen Salticidae.

The main aims of the present paper are (1) to describe a new Salticus species from Repetek, and (2) to publish new faunistic records of the jumping spiders from Turkmenistan based on old $(1985,1992,1993)$ collections of various people. An additional aim is to provide new faunistic records for the studied Turkmen salticids that have also been found in the neighbouring Central Asian countries, such as, Iran, Kyrgyzstan, Pakistan, Tajikistan and Uzbekistan.

\section{Material and Methods}

The studied specimens have been shared between the following museums and a private collection: AVG - personal collection A.V. Gromov, Almaty, Kazakhstan (will be later moved to the ZMMU); ISEA - Zoological Museum
Institute of Systematics and Ecology of Animals, Novosibirsk, Russia (curator: G.N. Azarkina); MMUE - the Manchester Museum, University of Manchester, UK (curator: D.V. Logunov); ZMMU - the Zoological Museum of the Moscow State University, Moscow, Russia (curator: K.G. Mikhailov).

The abbreviations used in the text: Eyes: AME - anterior median eye, PLE - posterior lateral eye(s). Leg segments: Fm - femur, $\mathrm{Pt}$ - patella, $\mathrm{Tb}$ - tibia, Mt metatarsus. For the leg spination the system adopted is that used by Ono [1988]. Other abbreviations: Distr. - district; nr. - near; Res. — reserve; Vil. — village. Collector names are abbreviated as follows: AAF - A.A. Fomichev; EAK E.A. Khachikov; SVO - S.V. Ovtchinnikov; VLP - V.L. Perepechaenko; EVP - E.V. Prokopenko. The sequence of leg segments in measurement data is as follows: femur + patella + tibia + metatarsus + tarsus. All measurements are in $\mathrm{mm}$. References to earlier taxonomic publications of the studied species are not provided and can be found in WSC [2019] and Metzner [2019]. 


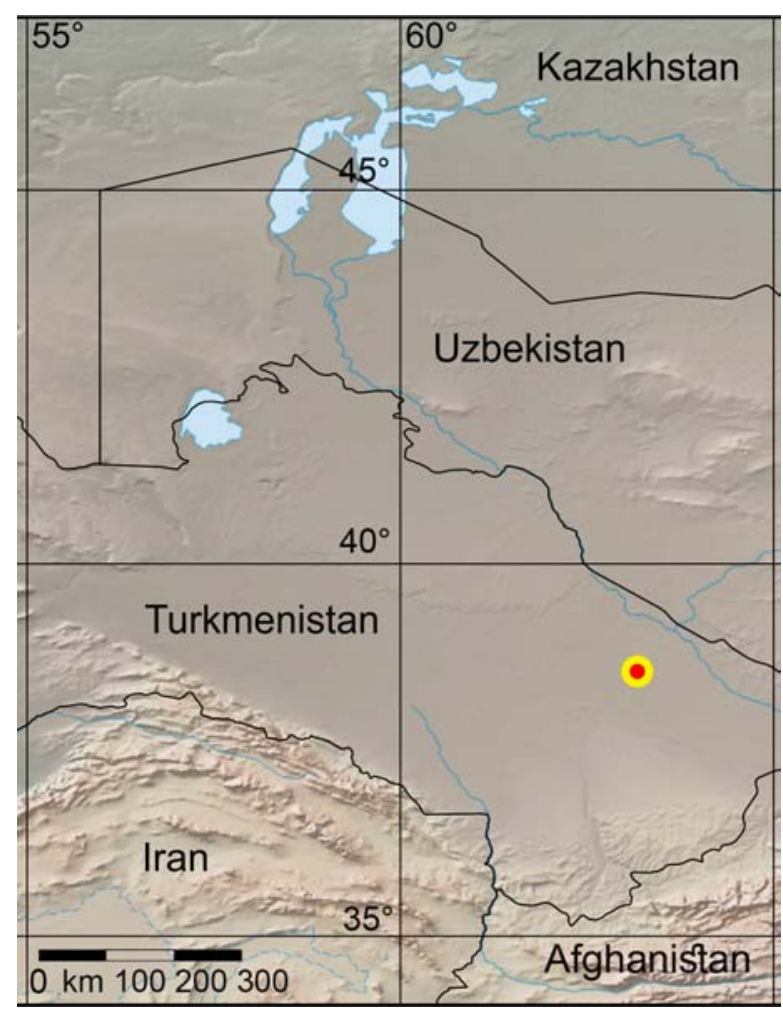

Map 1. The type locality of Salticus karakumensis sp.n.

Карта 1. Типовой локалитет Salticus karakumensis sp.n.

\section{Description}

\section{Salticus karakumensis sp.n.}

Figs 1-7, Map 1.

TYPE. Holotype $\sigma^{7}$ (ZMMU) from Turkmenistan, Repetek $\left(38^{\circ} 35^{\prime} 11^{\prime \prime} \mathrm{N}, 63^{\circ} 11^{\prime} 25^{\prime \prime} \mathrm{E}\right)$, Haloxylon desert woodland, 6.04.1993, EVP.

ETYMOLOGY. Named after the Karakum Desert where the type locality (Repetek Res.) is situated.

DIAGNOSIS. The male of $S$. karakumensis sp.n. (Figs $1,2)$ is closest to those of $S$. dzhungaricus Logunov, 1992 from Kazakhstan and S. turkmenicus Logunov et Rakov, 1998 from Turkmenistan (cf. figs 65-70 in Logunov \& Rakov [1998]), but differs from both in having the straight, wide and ribbon-shaped retrolateral tibial apophysis with an obtuse tip (bent ventrad in S. dzhungaricus and markedly pointed in S. turkmenicus) and different proportions of the bulbus $v s$. the embolus. The female of $S$. karakumensis sp.n. remains unknown, but see 'Comments' below.

COMMENTS. It is possible that the females of S. dzhungaricus reported by Logunov [1995a] and Logunov \& Rakov [1998] from Repetek and Chul'-Mamed'Kum sands are to be referred to $S$. karakumensis sp.n. The pertinent material of S. dzhungaricus studied by Logunov [1995a] and Logunov \& Rakov [1998] was inaccessible for the present study and therefore the latest assumption could not be verified. The matter requires a special attention in the future.

DISTRIBUTION. The type locality only (Map 1).

DESCRIPTION. Male. Carapace 1.58 long, 0.95 wide, 0.58 high at PLE. Ocular area 0.76 long, 0.84 wide anteriorly and 0.90 wide posteriorly. Diameter of AME 0.29. Abdo- men 1.80 long, 0.85 wide. Cheliceral length 0.57. Clypeus is not marked. Length of leg segments: I $0.75+0.48+0.55+$ $0.45+0.35$ (2.58); II $0.65+0.34+0.35+0.32+0.30$ $(1.96)$; III $0.65+0.33+0.43+0.40+0.35(2.16)$; IV $0.80+$ $0.33+0.53+0.53+0.38$ (2.57). Leg formula: I/IV,III,II. Legs spineless. Coloration (in alcohol; Figs 4-7); the specimen is in poor condition, shabby, with the carapace being detached from the abdomen. Carapace yellowish brown, with black eye field. Strenum brown. Endites and labium brown, with yellow apexes. Chelicerae yellowish brown; promargin with two small teeth, retromargin with a large tooth (Fig. 3). Entire abdomen yellowish brown, with no scale cover and colour pattern. Book-lung cover yellowish brown. Spinnerets grey-brown. Legs I: Fm brown, Pt and Tb dorsally and ventrally yellow, with both sides brown, Mt and Tr yellow. Legs II-III: Fm, Pt and Tb yellow, with brown patches and stripes, Mt and Tr yellow. Legs IV: Fm brown with dorsal yellow longitudinal stripe, $\mathrm{Pt}$ and $\mathrm{Tb}$ yellow, with both sides brown, Mt and $\mathrm{Tr}$ yellow. Palps yellow, their structure as in Figs 1, 2: RTA long, thick and wide; tegulum oval; embolus stiletto-like, bent retro-laterad, almost as long as RTA.

Female unknown. However, it is likely that the females of $S$. dzhungaricus reported by Logunov [1995a] and Logunov \& Rakov [1998] from Repetek and Chul'-Mamed'Kum sands are to be referred to $S$. karakumensis sp.n. (see above under 'Comments').

\section{New faunistic records}

\section{Aelurillus brutus Wesołowska, 1996}

MATERIAL. TURKMENISTAN: $1 \sigma^{7}$ (ZMMU), SW Kopetdagh, Syunt-Khasardagh Res. (c. $\left.38^{\circ} 32^{\prime} \mathrm{N}, 56^{\circ} 25^{\prime} \mathrm{E}\right), 14.03 .1993$, EVP; $1 \sigma^{7}$ (ZMMU), Magtymguly (=Kara-Kala) Distr., Balkan Velayat, c. $3 \mathrm{~km} \mathrm{~N}$ of Malyi Parkhai (c. $38^{\circ} 28^{\prime} 33^{\prime \prime}$ N, 56 $6^{\circ} 14^{\prime} 46^{\prime}$ 'E), Karaelchi Mt. Range, 11.12.1992, EVP; $1 \sigma^{\top}, 1$ \% (ZMMU), same distr. and velayat, outliers nr. Gyzylgaya $\left(40^{\circ} 37^{\prime} 17^{\prime \prime} \mathrm{N}, 55^{\circ} 28^{\prime} 00^{\prime \prime} \mathrm{E}\right)$, clay desert, 17.04.1993, EVP.

PREVIOUS RECORDS. South Ustyurt (Gaplaňgyr Res.) [Wesołowska, 1996], South Ustyurt (Butentau), Repetek [Azarkina, 2003].

DISTRIBUTION. Central Asian endemic, known from few localities in west and south-west Turkmenistan and west Kazakhstan [Azarkina, 2003: Fig. 15; present data].

\section{Aelurillus concolor Kulczyński, 1901}

MATERIAL. TURKMEnistan: 3 o (ZMMU), Magtymguly (=Kara-Kala) Distr., Balkan Velayat, c. $3 \mathrm{~km} \mathrm{~N}$ of Malyi Parkhai (c. $\left.38^{\circ} 28^{\prime} 33^{\prime \prime} \mathrm{N}, 56^{\circ} 14^{\prime} 46^{\prime \prime} \mathrm{E}\right)$, Karaelchi Mt. Range, 11-12.12.1992, VLP; $10^{7}, 3$ OO (ZMMU), same distr, and velayat, c. $3 \mathrm{~km}$ NW of Malyi Parkhai (c. $\left.38^{\circ} 28^{\prime} 33^{\prime \prime} \mathrm{N}, 56^{\circ} 14^{\prime} 46^{\prime \prime} \mathrm{E}\right)$, pedestal of Karaelchi Mt. Range, $500 \mathrm{~m}$ a.s.1., 13.12.1992, VLP; $1 \mathrm{\sigma}^{\top}$ (ZMMU), same distr. and velayat, c. $1 \mathrm{~km} \mathrm{~N}$ of Malyi Parkhai (c. $38^{\circ} 28^{\prime} 33^{\prime \prime} \mathrm{N}, 56^{\circ}$ 14'46"E), 11.12.1992, VLP; 1 丁 $^{\top}, 1$ (ZMMU), SW Kopetdagh, Syunt-Khasardagh Res. (c. $38^{\circ} 32^{\prime} \mathrm{N}, 5^{\circ} 25^{\prime} \mathrm{E}$ ), 7.03.1993, EVP.

PREVIOUS RECORDS. Chul'-Mamed'Kum sands, South Ustyurt (Gaplan̆gyr Res.) [Wesołowska, 1996], Magtymguly (=Kara-Kala), Parkhai, Firyuza (=Arçabyl), Ai-Dere, Tedzhenstroi (=Gaňňaly), c. $10 \mathrm{~km} \mathrm{SW}$ of Bäherden [Azarkina, Mirshamsi, 2014].

DISTRIBUTION. East-Mediterranean - Central Asian species, known from Greece in the west to Kyrgyzstan in the east, and south-eastward to Kerman Province of Iran [Azarkina, Mirshamsi, 2014: Fig. 1; present data]. 


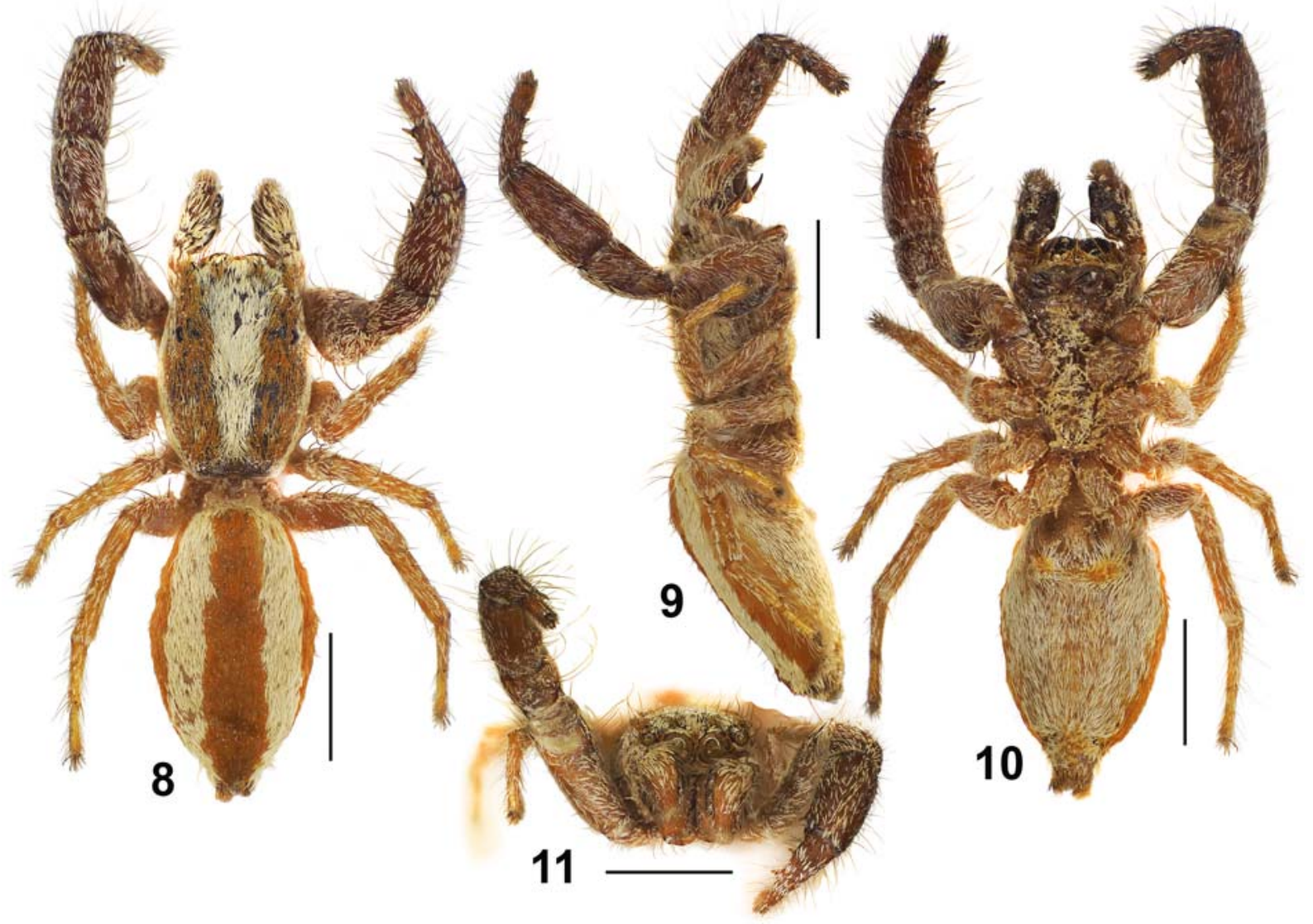

Figs 8-11. Male of Afraflacilla braunsi (Peckham et Peckham, 1903) from Charshanga, Turkmenistan: 8 - dorsal view; 9 - lateral view; 10 - ventral view; 11 - frontal view. Scale bars: $1 \mathrm{~mm}$.

Рис. 8-11. Самец Afraflacilla braunsi (Peckham et Peckham, 1903) из Чашанги, Туркменистан: 8 - вид сверху; 9 - вид сбоку; 10 - вид снизу; 11 - вид спереди. Масштаб: 1 мм.

\section{Aelurillus kopetdaghi Wesołowska, 1996}

MATERIAL. TuRKmenistan: 1 (ZMMU), Magtymguly (=Kara-Kala) Distr., Balkan Velayat, c. $3 \mathrm{~km} \mathrm{~N}$ of Malyi Parkha (c. $38^{\circ} 28^{\prime} 33^{\prime \prime} \mathrm{N}, 56^{\circ} 14^{\prime} 46^{\prime \prime} \mathrm{E}$ ), Karaelchi Mt. Range, 11.12.1992, VLP; $1 \sigma^{7}$ (ZMMU), c. $3 \mathrm{~km}$ NW of Malyi Parkhai (c. $38^{\circ} 28^{\prime} 33^{\prime \prime} \mathrm{N}$, $56^{\circ} 14^{\prime} 46^{\prime \prime} \mathrm{E}$ ), pedestal of Karaelchi Mt. Range, $500 \mathrm{~m}$ a.s.1., 13.12 . 1992, VLP; $2 \mathrm{O}^{7} \mathrm{O}^{7}$ (ZMMU), same distr. and velayat, c. $1 \mathrm{~km} \mathrm{~N}$ of Malyi Parkhai (c. $\left.38^{\circ} 28^{\prime} 33^{\prime \prime} \mathrm{N}, 56^{\circ} 14^{\prime} 46^{\prime \prime} \mathrm{E}\right), 11.12 .1992$, VLP; 2 $0^{7} \sigma^{\prime \prime}$ (ZMMU), same distr. and velayat, nr. Parkhai $\left(38^{\circ} 27^{\prime} 51^{\prime \prime} \mathrm{N}\right.$, $\left.56^{\circ} 14^{\prime} 58^{\prime \prime} \mathrm{E}\right), 11.12 .1992$, VLP; $3 \mathrm{O}^{7} \mathrm{O}^{7}, 5$ 우 (ZMMU), SW Kopetdagh, Syunt-Khasardagh Res. (c. $\left.38^{\circ} 32^{\prime} \mathrm{N}, 56^{\circ} 25^{\prime} \mathrm{E}\right), 7-14.03 .1993$, EVP. 1996].

PREVIOUS RECORDS. SW Kopetdagh [Wesołowska,

DISTRIBUTION. Central Asian endemic, known to date from SW Kopedagh only [Wesołowska, 1996; present data].

Afraflacilla braunsi (Peckham et Peckham, 1903) Figs 8-11.

MATERIAL. TURKMENISTAN: $2 \sigma^{7} \sigma^{7}$ (ZMMU), nr. Charshanga $\left(37^{\circ} 31^{\prime} 00^{\prime \prime} \mathrm{N}, 66^{\circ} 01^{\prime} 06^{\prime \prime} \mathrm{E}\right)$, saline clay desert, on Tamarix sp. 3.04.1993, VLP.

PREVIOUS RECORDS. Repetek, Tashauz [Logunov, 1995a; Wesołowska, 1996; both sub Pseudicius b.].

DISTRIBUTION. South Africa, Saudi Arabia, Yemen and Turkmenistan [Prószyński, 1989: sub Pseudicius tripunc- tata; Logunov, 1995a; Wesołowska, 1996; both sub Pseudicius b.].

COMMENTS. Here we have presented colour photographs of the male of this species (Figs 8-11) for the first time. The body colour and colour pattern of the studied male correspond and are complementary to the detailed description provided by Logunov [1995a: 240].

Afraflacilla flavipes (Caporiacco, 1935)

MATERIAL. TURKMENISTAN: 1 \% (ZMMU), Lebap Velayat, nr. Seidi $\left(39^{\circ} 29^{\prime} \mathrm{N}, 62^{\circ} 54^{\prime} \mathrm{E}\right)$, sand-clay desert, 12.04.1993, VLP; 1 우 (ZMMU), Amudariya Res. (c. $39^{\circ} 39^{\prime} \mathrm{N}, 62^{\circ} 51^{\prime} \mathrm{E}$ ), Kabakly site, tugai, 19.04.1993, VLP.

PREVIOUS RECORDS. Repetek [Logunov, Rakov, 1998: sub. Icius f.].

DISTRIBUTION. Central Asian endemic, known to date from four localities in Turkmenistan and Pakistan (Karakorum) only [Logunov, Rakov, 1998: sub. Icius f:; present data].

Chalcoscirtus infimus (Simon, 1868)

MATERIAL. Turkmenistan: 1 (ZMMU), Magtymguly (=Kara-Kala) Distr., Balkan Velayat, nr. Parkhai $\left(38^{\circ} 27^{\prime} 51^{\prime \prime} \mathrm{N}, 56^{\circ}\right.$ $\left.14^{\prime} 58^{\prime \prime} \mathrm{E}\right)$, foothills of Karaelchi Mt. Range, 2.05.1993, VLP. 
ADDITIONAL MATERIAL. KYRGYZSTAN: $3 \mathrm{O}^{7} \mathrm{O}^{7}$ (AVG), North Kyrgyzstan, Kirghizski Mt. Range, Ala-Archa River valley, Malinovka, c. $20 \mathrm{~km} \mathrm{~S}$ of Bishkek, 3.06.2004, SVO. UzBeKISTAN: 4 $\mathrm{O}^{7} \mathrm{O}^{7}$ (AVG), Babatagh Mt. Range, Ak-Mechet', 26.04.1994, SVO.

PREVIOUS RECORDS. SW Kopetdagh [Wesołowska, 1996], Kalaligyoz, Sumbar River, Magtymguly (=Kara-Kala), Firyuza (=Arçabyl), Kopetdagh Nature Res., Murgab, Morgunovka [Logunov, Marusik, 1999].

DISTRIBUTION. It is a common, Mediterranean - Central Asian species [Logunov, Marusik, 1999].

\section{Evarcha nenilini Rakov, 1997}

Figs 12-19.

TYPE. Holotype $\sigma^{7}$ (ISEA, SZM 000.087) from [Uzbekistan], "9.05.1976, Bukantau, Irlir, Karakud well, A.P. Kononenko" (Figs 16-19). - In the original publication [Rakov, 1997: 110], a slightly different label is given: "Uzbekistan, Bukantau Mts., Irmir Mt., Karakuduk"; the rough coordinates for Karakuduk are as follow: $40^{\circ} 46^{\prime} \mathrm{N}, 69^{\circ} 14^{\prime \prime} \mathrm{E}$.

MATERIAL. TURKMENISTAN: $10^{7}$ (ZMMU), SW Kopetdagh, Syunt-Khasardagh Res. (c. $38^{\circ} 32^{\prime} \mathrm{N}, 56^{\circ} 25^{\prime} \mathrm{E}$ ), 14.03.1993, EVP.

DISTRIBUTION. Central Asian endemic, known to date from six localities only [Rakov, 1997; present data]. New to Turkmenistan.

COMMENTS. This species was recently transferred to a separate genus Evaneg Prószyński, 2018 encompassing 21 species (see Prószyński [2018]). However, the latter generic name turned out to be a subjective junior synonym of Colopsus Simon, 1902 [Blick, Marusik, 2018]. To date, neither the generic name Evaneg, nor the combination Colopsus nenilini has been officially accepted by the WSC [2019]; see Kropf et al. [2019] for the justification. Thus, in this paper we follow the nomenclature suggested by the WSC [2019] and consider this species in Evarcha Simon, 1902 (s. lat.).

The studied male is slightly bigger (c. 23\%) than the holotype male of E. nenilini, but is identical to it by its body coloration (cf. Figs 12-15 and 16-19). The general appearance of the holotype was not shown in the original description and hence is illustrated herein for the first time. 1872)

Heliophanus curvidens (O. Pickard-Cambridge,

MATERIAL. TURKMENISTAN: $1 \sigma^{\top}$ (ZMMU), Charshanga Distr., Lebap Velayat, c. $2 \mathrm{~km}$ E of Bazar-Depe kishlak (c. $37^{\circ} 48^{\prime} \mathrm{N}$, $66^{\circ} 30^{\prime}$ E), Kugitangh Res., low-mountain relief, 29.03.1993, EVP.

ADDITIONAL MATERIAL. TAJIKISTAN: $1 \mathrm{O}^{\text {T }}$ (ISEA; det. G.N Azarkina), Khatlon Area, Gardaniushti Mt. Range, c. 2 km NW of Dakhanakiik kishlak $\left(38^{\circ} 12.883^{\prime} \mathrm{N}, 68^{\circ} 36.861^{\prime} \mathrm{E}\right)$, dry stony scrubland vegetation with rocks, $1130-1250 \mathrm{~m}$ a.s.1., 20.04.2019, AAF; $1 \mathrm{O}^{7}, 1$ (ISEA; det. G.N. Azarkina), same area, Dzhilantau Mt. Range, c. 2 km E of Kul'bul'on kishlak $\left(37^{\circ} 53.970^{\prime} \mathrm{N}, 69^{\circ} 23.155^{\prime} \mathrm{E}\right)$, dry stony scrubland vegetation with rocks, $970-1200 \mathrm{~m}$ a.s.l., 14.04.2019, AAF

PREVIOUS RECORDS. Bolshoy Balkhan Mts [Wesołowska, 1996], Zhdanova Kolkhoz, c. $10 \mathrm{~km} \mathrm{~S}$ of Bäherden, Badkhyz Res., Magtymguly (=Kara-Kala), Bolshoi Balkhan Mts., Zul'fagar Mt. Range, Gezgyadyk Mt. Range, Firyuza (=Arçabyl), Kugitangtau Mt. Range (Airi-Baba Mt.), KaraBelent Mts, Basar-Tepe, Khodzhapil [Rakov, Logunov, 1997a].

DISTRIBUTION. It is a common species known from the Middle East, throughout Iran to western China (Gansu), Pakistan (Karakorum) and Mongolia in the east [Rakov, Logunov, 1997a; Logunov et al., 2007].

\section{Heliophanus forcipifer Kulczyński, 1895}

MATERIAL. TURKMENISTAN: $2 \sigma^{7} \sigma^{7}, 1$ ( 1 (ZMMU), Amudariya Res. (c. $\left.39^{\circ} 39^{\prime} \mathrm{N}, 62^{\circ} 51^{\prime} \mathrm{E}\right)$, Kabakly site, tugai, 19.04.1993, VLP.

DISTRIBUTION. The Caucasus, Iran and Central Asia [Rakov, Logunov, 1997a; Logunov et al., 2002]. New to Turkmenistan.

Heliophanus mordax (O. Pickard-Cambridge, 1872)

MATERIAL. TURKMENISTAN: $1 \overbrace{}^{\nearrow,} 2$ † (ZMMU), Magtymguly (=Kara-Kala) Distr., Balkan Velayat, nr. Parkhai $\left(38^{\circ} 27^{\prime} 51^{\prime \prime} \mathrm{N}\right.$, $56^{\circ} 14^{\prime} 58^{\prime \prime} \mathrm{E}$ ), foothills of Karaelchi Mt. Range, 2-4.05.1993, VLP, EVP; 1 \& (ZMMU), same distr. and velayat, Shikhin-Dere valley $\left(38^{\circ} 27^{\prime} 14^{\prime \prime} \mathrm{N}, 56^{\circ} 16^{\prime} 07^{\prime \prime} \mathrm{E}\right)$, foothills of Karaelchi Mt. Range, 6.05 . 1993, VLP; 2 O$^{7} 0^{7}, 1$ q (ZMMU), SW Kopetdagh, Syunt-Khasardagh Res. (c. $\left.38^{\circ} 32^{\prime} \mathrm{N}, 56^{\circ} 25^{\prime} \mathrm{E}\right), 14.03 .1993$, EVP.

PREVIOUS RECORDS. C and SW Kopetdagh [Wesołowska, 1996], Aidere, Parkhai, S sloping of Isak Mt., Sumbar River, Firyuza (=Arçabyl), Karaelchi Canyon, Morgunovka [Rakov, Logunov, 1997a], Malyi Balkhan [Logunov, Marusik, 2000a]

DISTRIBUTION. An eastern Mediterranean - Central Asian species, known from Greece and Egypt in the west, throughout Asia Minor, the Near East and the Caucasus, eastward to south-west and central Kopetdagh in Turkmenistan and Khorasan province of Iran [Logunov, 2015; present data].

\section{Heliophanus turanicus Charitonov, 1969}

MATERIAL. TURKMENISTAN: 1 (ZMMU), SW Kopetdagh, Syunt-Khasardagh Res. (c. $38^{\circ} 32^{\prime} \mathrm{N}, 56^{\circ} 25^{\prime} \mathrm{E}$ ), Sumbar River valley, litter under Caragana bushes, 14.03.1993, EVP.

ADDITIONAL MATERIAL. TAJIKISTAN: 19 (ISEA; det. G.N. Azarkina), Khatlon Area, Dzhilantau Mt. Range, c. $2 \mathrm{~km} \mathrm{E}$ of Kul'bul'on kishlak $\left(37^{\circ} 53.970^{\prime} \mathrm{N}, 69^{\circ} 23.155^{\prime} \mathrm{E}\right)$, dry stony scrubland vegetation with rocks, 970-1200 m a.s.1., 14.04.2019, AAF; $2 \bigcirc^{7} O^{7}$ (ISEA; det. G.N. Azarkina), same area, Shurobdariya River valley, Bal'dzhuvon kishlak $\left(8^{\circ} 18.835^{\prime} \mathrm{N}, 69^{\circ} 41.011^{\prime} \mathrm{E}\right)$, stony-clay slopes with meadow vegetation, $900-1000 \mathrm{~m}$ a.s.1., 15.04.2019, AAF.

PREVIOUS RECORDS. Badkhyz Res., Karatau Mt. Range (Astana Mt.), Zulfagarskiy Mt. Range, Gezgyadyk Mt. Range, Kala-i-Mor, Morgunovka, Chemen-Ibit, NebitDag, Magtymguly (=Kara-Kala) [Rakov, Logunov, 1997a].

DISTRIBUTION. Central Asian (Turanian) endemic [Rakov, Logunov, 1997a; present data].

\section{Langona pallidula Logunov et Rakov, 1998} Figs 20-22, Map 2.

MATERIAL. TURKMENISTAN: $1 \mathrm{O}^{7}$ (ZMMU), $70-80 \mathrm{~km} \mathrm{~N}$ of Turkmenbashi (=Krasnovodsk), Chul'-Mamed'Kum sands $\left(40^{\circ}\right.$ $\left.35^{\prime} \mathrm{N}, 53^{\circ} 05^{\prime} \mathrm{E}\right)$, nr. Sofi $\left(2 \mathrm{~km}-270^{\circ}\right), 15.05 .1985$, EAK. 1998].

PREVIOUS RECORDS. Repetek [Logunov, Rakov,

DISTRIBUTION. To date, the species is known from three localities in Turkmenistan and Iran only (Map 2) [Logunov, Rakov, 1998; Logunov et al., 2013; Azarkina, Zamani, 2019; present data].

COMMENTS. Despite the examined male is in a rather poor condition, it has been possible to confirm the diagnosis of L. pallidula from the second Middle Asian Langona species L. tartarica (see Logunov \& Rakov [1998]), from which it can easily be distinguished by the densely white haired clypeus and chelicerae (Fig. 21) (chelicerae dark 
D.V. Logunov, A.V. Ponomarev

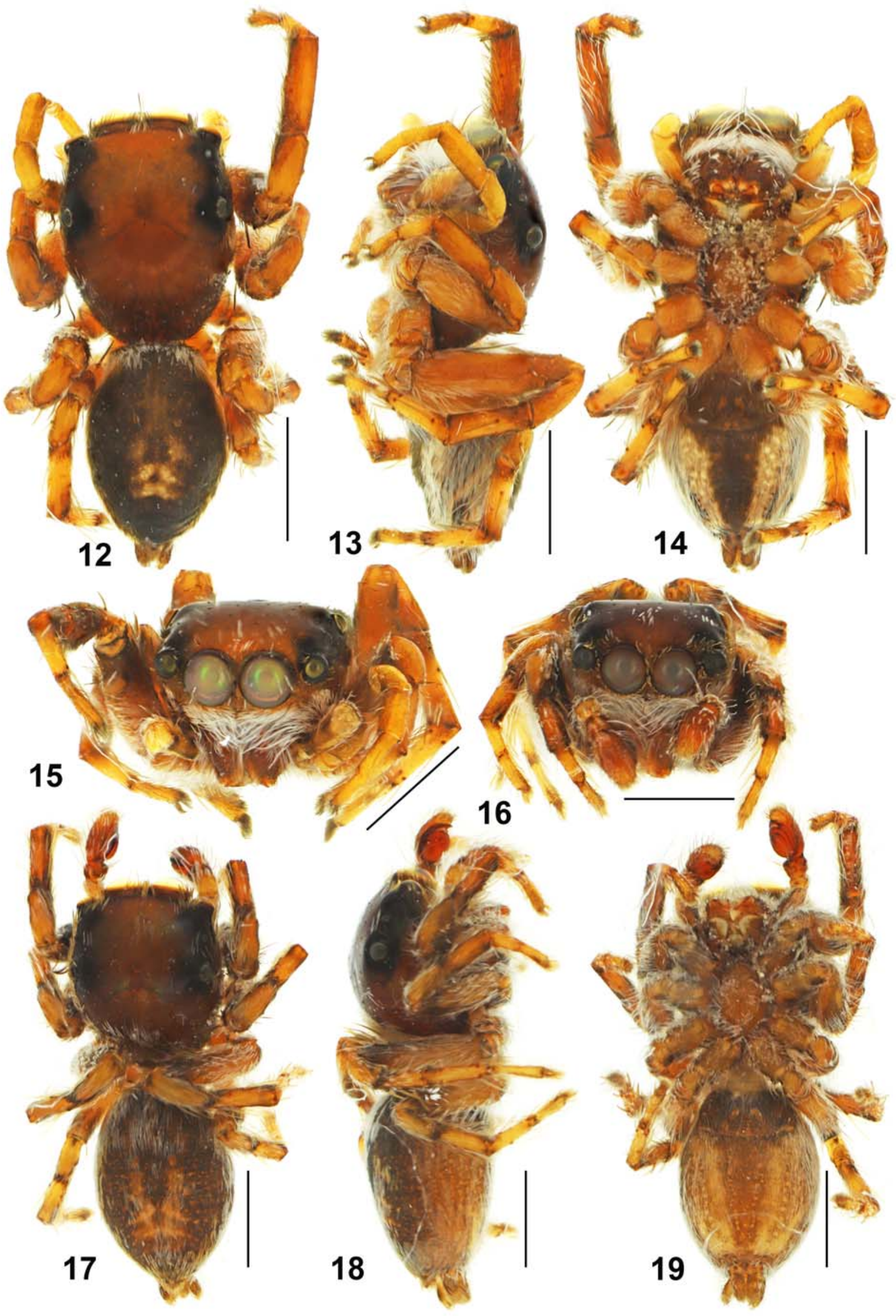




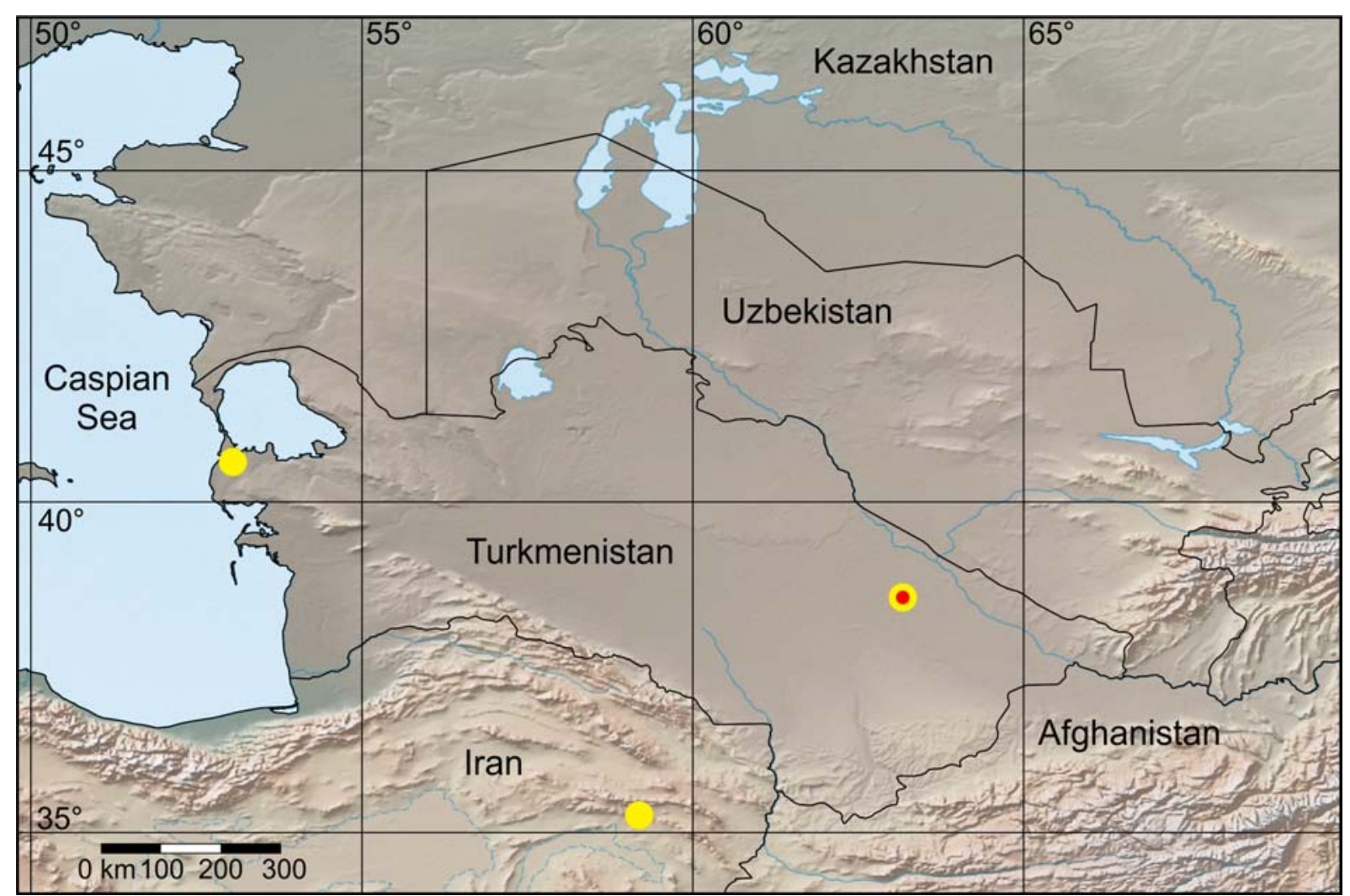

Map 2. Collecting localities of Langona pallidula Logunov et Rakov, 1998; the type locality is marked with red spot. Карта 2. Точки сбора Langona pallidula Logunov et Rakov, 1998; типовой локалитет помечен красной точкой.

brown, with dark brow hairs in their distal two-thirds in $L$. tartarica; Fig. 24), the femora I without a longitudinal dark brown stripe (Fig. 22) (present in L. tartarica; Fig. 25), and the dorsum without a clearly-marked colour pattern (Fig. 20) (with two longitudinal wide brown stripes in L. tartarica; Fig. 23). Yet, the taxonomic status of L. pallidula itself is to be further revised when more specimens of both sexes have been collected, particularly in relation to two closely related but poorly diagnosed Langona species recorded from Afghanistan and Iran: viz., L. aperta (Denis, 1958) and $L$. redii (Audouin, 1826) [Logunov, Zamanpoore, 2005; Logunov et al., 2002, 2013; Azarkina, Zamani, 2019]. The entire and very difficult genus Langona Simon, 1901 is in need of an urgent and thorough revision.

\section{Langona tartarica (Charitonov, 1946)} Figs 23-25.

MATERIAL. TURKMENISTAN: $1 \sigma^{7}$ (ZMMU), Charshanga Distr., Lebap Velayat, c. $3 \mathrm{~km}$ E of Bazar-Depe kishlak (c. $37^{\circ} 48^{\prime} \mathrm{N}$, $66^{\circ} 30^{\prime} \mathrm{E}$ ), Kugitangh Res., low-mountain relief, 31.03.1993, VLP; $10^{7}, 1$ \& (ZMMU), nr. Charshanga $\left(37^{\circ} 31^{\prime} 00^{\prime \prime} \mathrm{N}, 66^{\circ} 01^{\prime} 06^{\prime \prime} \mathrm{E}\right)$, saline clay desert, on Tamarix sp., 3.04.1993, VLP.

ADDITIONAL MATERIAL. PAKistan: $1 \sigma^{7}, 1+$ (ZMMU), 1 $\mathrm{O}^{7}, 1$ (MMUE), NWF, Peshawar, Forestry Campus of Agricultur- al University, 4.09.2004, SVO; $2 \sigma^{\top} \sigma^{7}$ (MMUE), Karachi, no date, M. Kazim.

PREVIOUS RECORDS. SW Kopetdagh, Farab, Kabakly [Wesołowska, 1996], Ashgabat, Kizil-Arvat, Morgunovka, Sultanbent, Murghab riverside, Turkmenbashi (=Krasnovodsk), Firyuza (=Arçabyl), Bazar-Depe [Logunov, Rakov, 1998].

DISTRIBUTION. The species is known from Central Asia only [Logunov, Rakov, 1998], with the south-westernmost locality lying in Iran [Logunov et al., 2007]. The record of this species from Yemen [Wesołowska, van Harten, 2007] was based on the females, which are virtually impossible to distinguish between Langona species, and needs confirmation by a corresponding male collected from that area. New to Pakistan.

\section{Marpissa nivoyi (Lucas, 1846)}

MATERIAL. TURKMENISTAN: 1 ? (ZMMU), Amudariya Res. (c. $\left.39^{\circ} 39^{\prime} \mathrm{N}, 62^{\circ} 51^{\prime} \mathrm{E}\right)$, Kabakly site, tugai, 19.04 .1993 , VLP.

ADDitional MATERIAL. Kyrgyzstan: $10^{7}$ (AVG), S of Bishkek, park zone, 9.06.1998, SVO.

DISTRIBUTION. It is a European - Central Asian species, known from Morocco and Algeria [Logunov, 2015], eastward to south Kazakhstan and Kyrgyzstan [Logunov,

Figs 12-19. Evarcha nenilini Rakov, 1997, male from Syunt-Khasardagh Res., Turkmenistan (12-15) and the holotype (16-19): 12, 17 - general appearance, dorsal view; 13, 18 - ditto, lateral view; 14, 19 - ditto, ventral view; 15, 16 - ditto, frontal view. Scale bars: $1 \mathrm{~mm}$.

Рис. 12-19. Evarcha nenilini Rakov, 1997, самец из Сюнт-Хасардагского запов., Туркменистан (12-15) и голотип (16-19): 12, 17 - общий вид, сверху; 13, 18 - тоже, сбоку; 14, 19 - тоже, снизу; 15, 16 - тоже, спереди. Масштаб: 1 мм. 


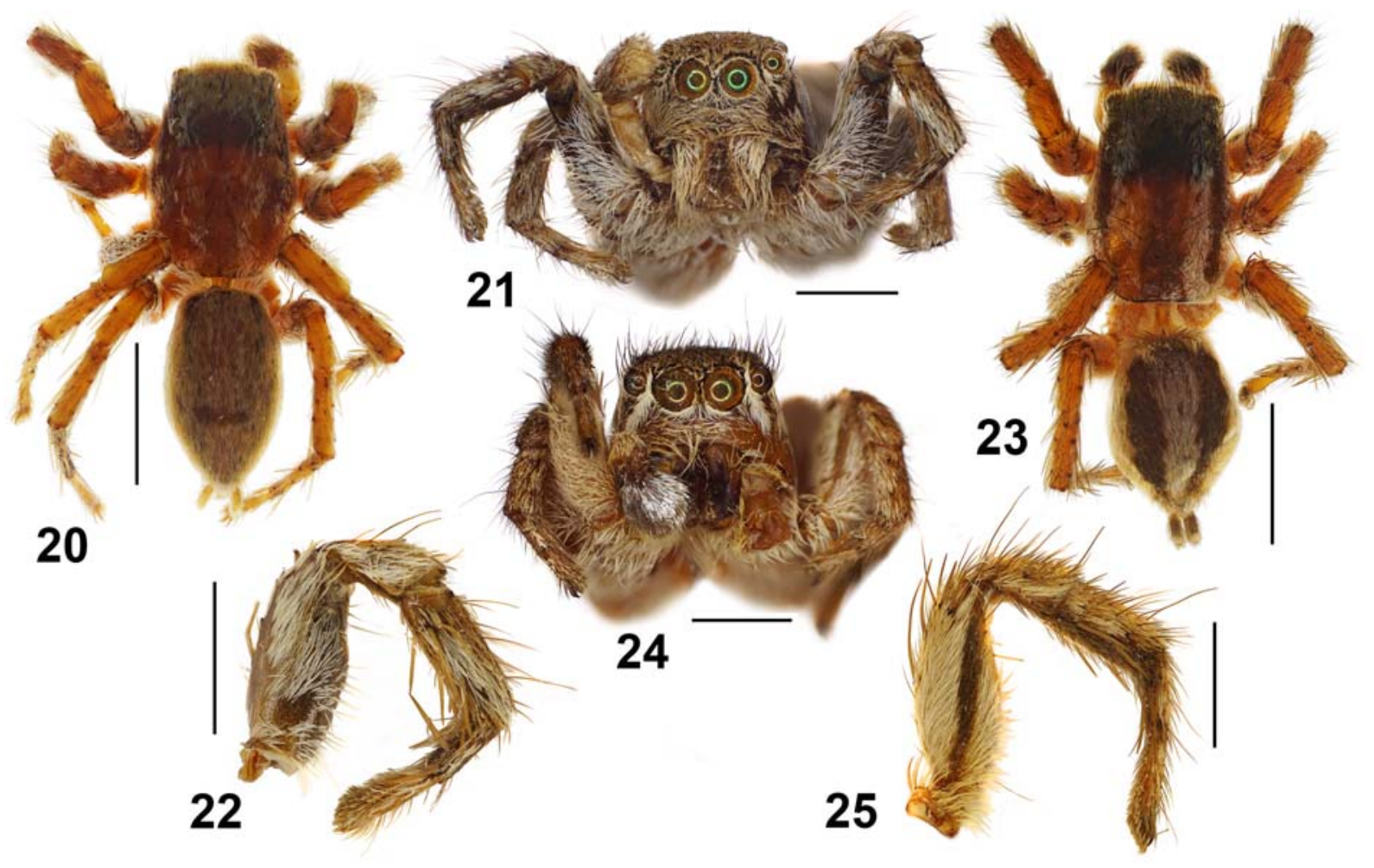

Figs 20-25. Males of Langona pallidula Logunov et Rakov, 1998 from Chul'-Mamed'Kum sands, Turkmenistan (20-22) and $L$. tartarica (Charitonov, 1946) from Charshanga, Turkmeninstan (23-25): 20, 23 - general appearance, dorsal view; 21, 24 - ditto, frontal view; 22, 25 - leg I, frontal view. Scale bars: $1 \mathrm{~mm}$.

Рис. 20-25. Самцы Langona pallidula Logunov et Rakov, 1998 из песков Чулмамедкум, Туркменистан (20-22) и L. tartarica (Charitonov, 1946) из Чаршанги, Туркменистан (23-25): 20, 23 - общий вид, сверху; 21, 24 - тоже, спереди; 22, 25 — нога I, вид спереди. Масштаб: 1 мм.

Rakov, 1998], southward to Iran [Logunov, 2010]. New to Turkmenistan.

Marusyllus aralicus (Logunov et Marusik, 2003)

MATERIAL. TURKMENISTAN: $1 \overbrace{}^{7}, 1$ (ZMMU), 70-80 km N of Turkmenbashi (=Krasnovodsk), Chul'-Mamed'Kum sands ( $40^{\circ}$ $\left.35^{\prime} \mathrm{N}, 53^{\circ} 05^{\prime} \mathrm{E}\right)$, nr. Adzhikuyu, 25-30.05.1985, EAK.

PREVIOUS RECORDS. Kankakyr Height, Repetek, Eradzi sands, Kizyl-Su [Logunov, Marusik, 2003: sub Yllenus a.].

DISTRIBUTION. It is a lowland Turanian species, known from Azerbaijan (Absheron Peninsula), south-western Kazakhstan and Turkmenistan only [Logunov, Marusik, 2003: Map 27; sub Yllenus a.]. 2003)

Marusyllus uzbekistanicus (Logunov et Marusik,

MATERIAL. TURKMENISTAN: 4 ㅇ (ZMMU), Repetek ( $38^{\circ} 35^{\prime}$ $\left.11^{\prime \prime} \mathrm{N}, 63^{\circ} 11^{\prime} 25^{\prime \prime} \mathrm{E}\right), 8.04 .1993$, VLP.

PREVIOUS RECORDS. Repetek, Shakhsenem well, Lake Sarykamysh, Amudariya Res., Uch-Adzhi, Uzboi [Logunov, Marusik, 2003: sub Yllenus u.].

DISTRIBUTION. It is a lowland Turanian species, known from West Kazakhstan, Turkmenistan and Uzbekistan only [Logunov, Marusik, 2003: Map 28; sub Yllenus u.; Ponomarev, 2008: sub Yllenus u.].

\section{Menemerus marginatus (Kroneberg, 1875)}

MATERIAL. Turkmenistan: $1 \sigma^{\top}$ (ZMMU), Amudariya Res. (c. $39^{\circ} 39^{\prime} \mathrm{N}, 62^{\circ} 51^{\prime} \mathrm{E}$ ), Kabakly site, tugai, 19.04.1993, VLP.

ADDITIONAL MATERIAL. PAKISTAN: 1 (MMUE), Faisarabad, barren ground, 21.09.2004, A. Butt; $1 \sigma^{7}, 1$ ( (ZMMU), NWF, Peshawar, Forestry Campus of Agricultural University, 26-29.08. 2004, SVO; 2 O $^{\top}$ Oొ $^{7}$ (MMUE), Karachi, 7.02-13.03.2013, M. Kazim.

PREVIOUS RECORDS. SW Kopetdagh, Amudariya Res., Badkhyz, Yashlyk, Repetek, Sultanbent, Ashgabad [Wesołowska, 1996; Rakov, Logunov, 1997b].

DISTRIBUTION. The species is known from the United Arab Emirates [Wesołowska, van Harten, 2011], throughout Iran [Logunov et al., 2007; Mirshamsi et al., 2013] and the eastern Caucasus (Azerbaijan and Chechnya) [Rakov, Logunov, 1997b] to Tajikistan, Afghanistan and Pakistan in the east [Rakov, Logunov, 1997b; Logunov, Zamanpoore, 2005; Bauer et al., 2015; present data].

\section{Mogrus antoninus Andreeva, 1976}

MATERIAL. TURKMENISTAN: 1 (ZMMU), $70-80 \mathrm{~km} \mathrm{~N}$ of Turkmenbashi (=Krasnovodsk), Chul'-Mamed'Kum sands $\left(40^{\circ}\right.$ $35^{\prime} \mathrm{N}, 53^{\circ} 05^{\prime} \mathrm{E}$ ), nr. Tezet-Kuyu well, 18.05.1985, EAK; 1 우 (ZMMU), Magtymguly (=Kara-Kala) Distr., Balkan Velayat, nr. Parkhai $\left(38^{\circ} 27^{\prime} 51^{\prime \prime} \mathrm{N}, 56^{\circ} 14^{\prime} 58^{\prime \prime} \mathrm{E}\right)$, foothills of Karaelchi Mt. Range, 2.05.1993, VLP, EVP. 


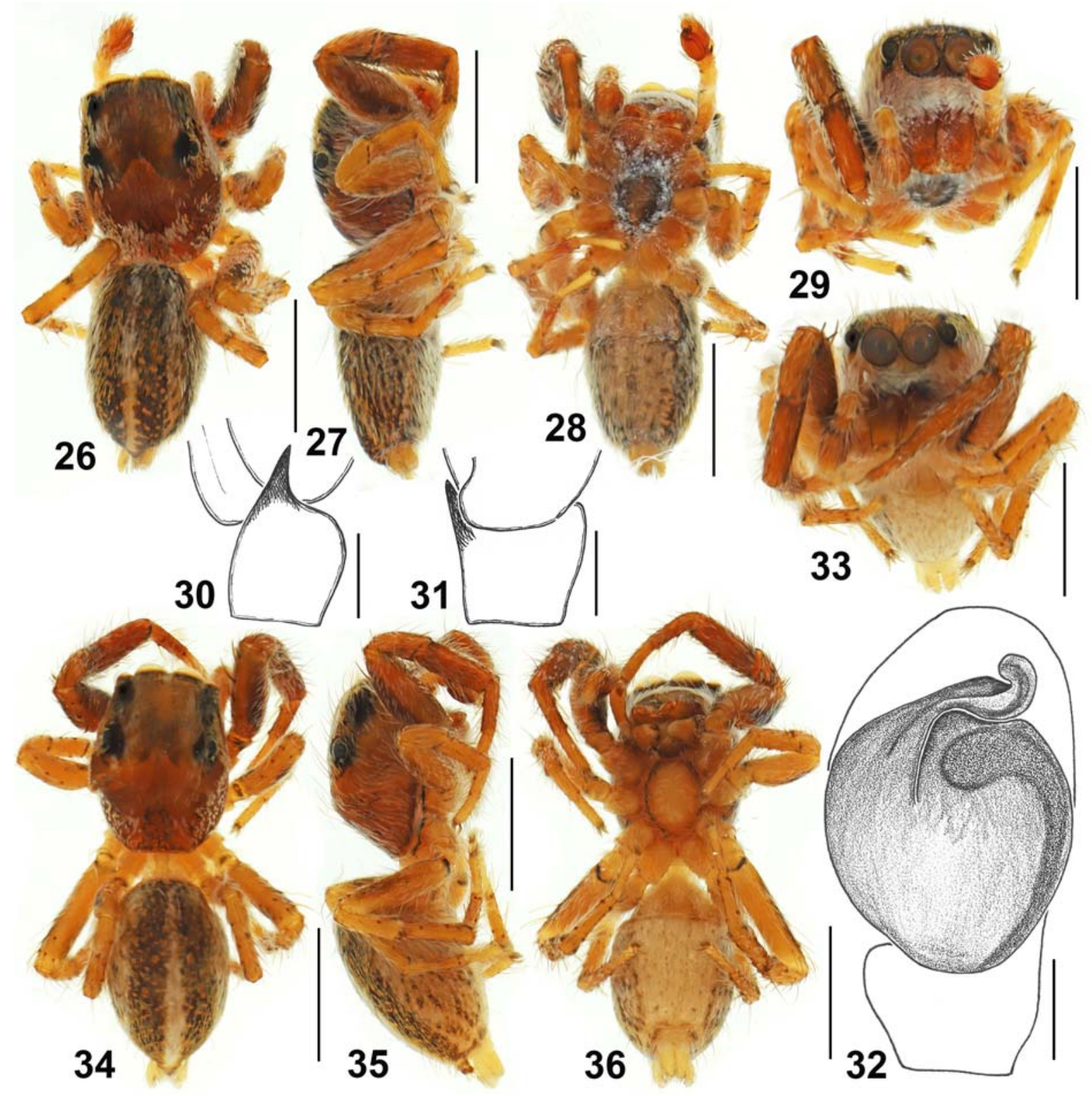

Figs 26-36. Pellenes sytchevskayae Logunov, Marusik et Rakov, 1999, male from Gyzylgaya, Turkmenistan (26-32) and the holotype (33-36): 26, 34 - general appearance, dorsal view; 27, 35 - ditto, lateral view; 28, 36 - ditto, ventral view; 29, 33 - ditto, frontal view; 30 - retrolateral tibial apophysis, lateral view; 31 - ditto, dorsal view; 32 - male palp, ventral view. Scale bars: (30-32) $0.1 \mathrm{~mm},(26-$ 29, 33-36) $1 \mathrm{~mm}$.

Рис. 26-36. Pellenes sytchevskayae Logunov, Marusik et Rakov, 1999, самец из Гызылгая, Туркменистан (26-32) и голотип (3336): 26, 34 - общий вид, сверху; 27, 35 - тоже, сбоку; 28, 36 - тоже, снизу; 29, 33 - тоже, спереди; 30 - ретролатеральный тибиальный отросток, сбоку; 31 - тоже, сзади; 32 - пальпа самца, вид снизу. Масштаб: (30-32) 0,1 мм, (26-29, 33-36) 1 мм.

PREVIOUS RECORDS. Tashauz, Kelif, Magtymguly (=Kara-Kala), Sumbar River [Logunov, 1995b], Amudariya Res., Sultanbent, South Ustyurt (Gaplaňgyr Res.) [Wesołowska, 1996].

DISTRIBUTION. This seems to be a Central Asian endemic known from Iran (Razavi Khorasan) in the south-west [Mirshamsi et al., 2013; Namaghi et al., 2016] to NW China (Xinjiang) in the north-east [Logunov, 1995b: Fig. 17].
Pellenes (Pelmirus) sytchevskayae Logunov, Marusik et Rakov, 1999

Figs 26-36, Map 3.

TYPE. Holotype $\sigma^{7}$ (ISEA, SZM 000.373; Figs 33-36) from Uzbekistan, Bukhara Area, c. $1 \mathrm{~km}$ NE of Gazli $\left(40^{\circ} 07^{\prime} 52^{\prime \prime} \mathrm{N} 63^{\circ}\right.$ $27^{\prime} 22^{\prime \prime}$ E), 15.05.1994, A.A. Zyuzin. 


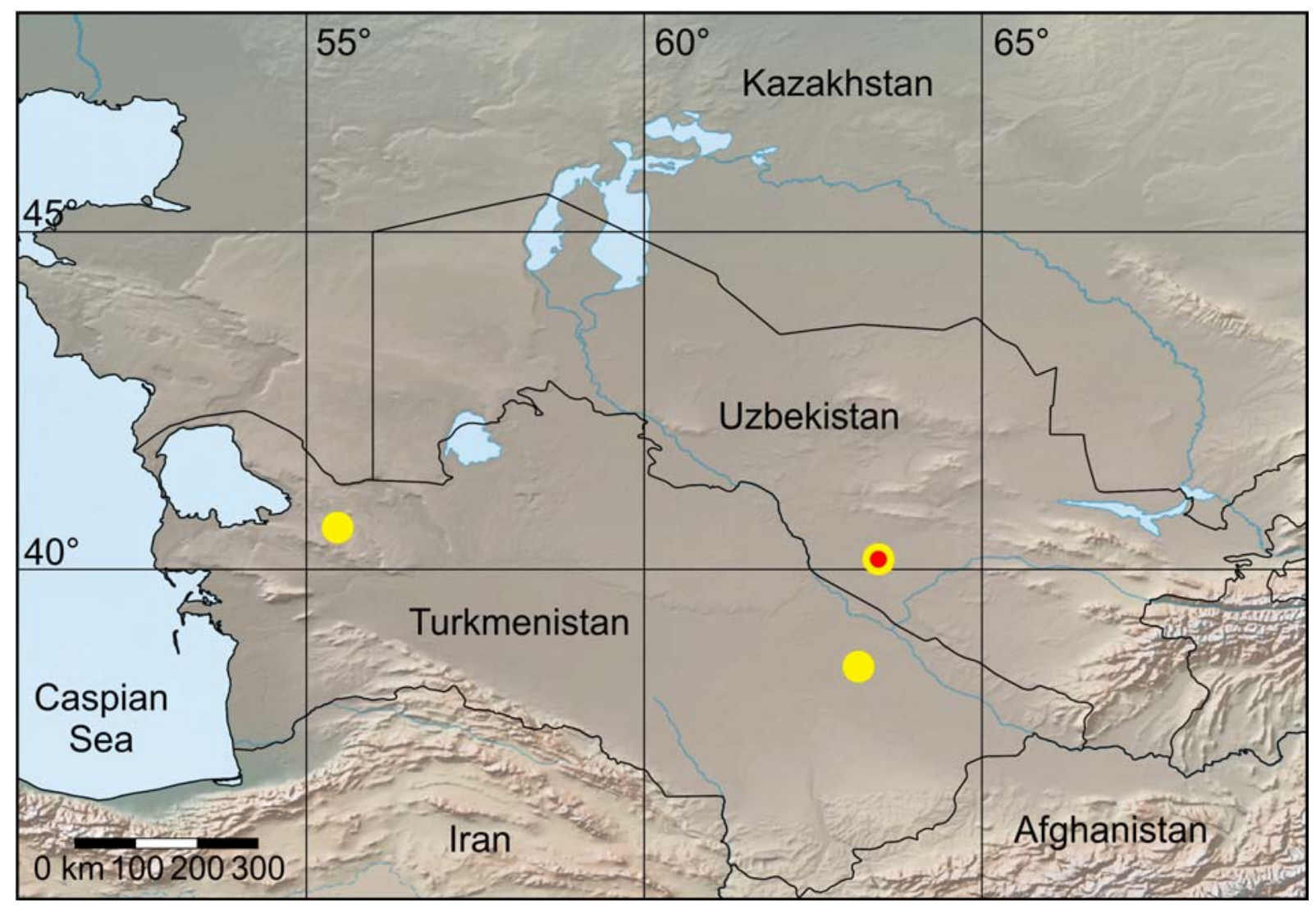

Map 3. Collecting localities of Pellenes sytchevskayae Logunov, Marusik et Rakov, 1999; the type locality is marked with red spot.

Карта 3. Точки сбора Pellenes sytchevskayae Logunov, Marusik et Rakov, 1999; типовой локалитет помечен красной точкой.

MATERIAL. Turkmenistan: 1 or (ZMMU), Magtymguly (=Kara-Kala) Distr., Balkan Velayat, nr. Gyzylgaya $\left(40^{\circ} 37^{\prime} 17^{\prime \prime} \mathrm{N}\right.$, $55^{\circ} 28^{\prime} 00^{\prime \prime} \mathrm{E}$ ), outliers in clay desert, 17.04.1993, EVP.

PREVIOUS RECORDS. Repetek [Logunov et al., 1999].

DISTRIBUTION. Central Asian (Turanian) endemic, known to date from three localities only (Map 3) [Logunov et al., 1999; present data].

COMMENTS. The studied Turkmen male is virtually identical to the holotype, both in its body size and coloration (cf. Figs 26-29 and 33-36) and in the conformation of male palp (cf. Figs 30-32 and figs 53-55 in Logunov et al. [1999]). The only tiny difference is seen in the sternum coloration, which is dark brown in the Turkmen male (Fig. 28) and yellow with a thin brown piping in the holotype (Fig. 36); it is hardly more than a variation. Logunov et al. [1999] provisionally matched the sexes of P. sytchevskayae, which originated from two close localities: viz., the holotype male from Gazli (Bukhara Area, Uzbekistan) and the female paratype from Repetek (Turkmenistan). The matter remains unresolved until both sexes are collected together.

\section{Pellenes (Pelmultus) allegrii Caporiacco, 1935}

MATERIAL. Turkmenistan: 19 (ZMMU), Magtymguly (=Kara-Kala) Distr., Balkan Velayat, nr. Parkhai $\left(38^{\circ} 27^{\prime} 51^{\prime \prime} \mathrm{N}, 56^{\circ}\right.$ $\left.14^{\prime} 58^{\prime \prime} \mathrm{E}\right)$, foothills of Karaelchi Mt. Range, 2.05.1993, VLP; $1 \sigma^{7}$ (ZMMU), SW Kopetdagh, Syunt-Khasardagh Res. (c. $38^{\circ} 32^{\prime} \mathrm{N}$, $\left.56^{\circ} 25^{\prime} \mathrm{E}\right), 14.03 .1993$, EVP.

DISTRIBUTION. The species is currently known from the south part of European Russia (from Krasnodar Territo- ry and Rostov Area [Ponomarev, Prishutova, 2017; Ponomarev et al., 2018] throughout Stavropol Territory and Dagestan [Ponomarev, 2008; Ponomarev et al., 2017] to Kalmykia and Volgograd Area [Ponomarev, Khnykin, 2013]), south-west Turkmenistan [present data], east Kazakhstan, east Uzbekistan and Kyrgyzstan [Logunov et al., 1999]. New to Turkmenistan.

\section{Pellenes (Pelmultus) epularis (O. Pickard-Cam-} bridge, 1872)

MATERIAL. TURKMENISTAN: $4 \sigma^{\top} \sigma^{\top}, 1$ (ZMMU), nr. Charshanga $\left(37^{\circ} 31^{\prime} 00^{\prime \prime} \mathrm{N}, 66^{\circ} 01^{\prime} 06^{\prime \prime} \mathrm{E}\right)$, saline clay desert, on Tamarix sp., 3.04.1993, VLP.

ADDITIONAL MATERIAL. KYRGYZSTAN: 1 (AVG), Baubashata Mt. Range, Arslanbob, 19.05.1993, SVO. UzBeKISTAN: 1 o (AVG), Zeravshan Mt. Range, Aman-Kutan pass, 27.04.1993, SVO.

PREVIOUS RECORDS. Murghab [Prószyński, 1979: sub Pellenes sp.-2], Chemen-Ibit, Dushak Mt., Germab, Saptykachi, Magtymguly (=Kara-Kala) [Logunov et al., 1999].

DISTRIBUTION. It is a common Central Asian species known from Iran and the Caucasus in the west [Logunov et al., 1999, 2002; Zamani et al., 2019] across Central Asia to Kyrgyzstan and Tajikistan in the east [Logunov et al., 1999].

Pellenes (Pelmultus) geniculatus (Simon, 1868)

MATERIAL. Turkmenistan: $1 \sigma^{7}$ (ZMMU), Charshanga Distr., Lebap Velayat, c. $3 \mathrm{~km}$ E of Bazar-Depe kishlak (c. $37^{\circ} 48^{\prime} \mathrm{N}$, $66^{\circ} 30^{\prime} \mathrm{E}$ ), Kugitangh Res., low-mountain relief, 31.03.1993, VLP; 
$1 \mathrm{O}^{7}$ (AVG), SW Kopetdagh, Magtymguly (=Kara-Kala) (c. $38^{\circ} 26^{\prime} \mathrm{N}$ $\left.56^{\circ} 17^{\prime} \mathrm{E}\right), 27.03 .1993$, SVO; $2 \mathrm{O}^{7} \mathrm{O}^{7}$ (AVG), Zulfagar Mt. Range, c. $37 \mathrm{~km}$ SE of Pulikhatum (c. $\left.35^{\circ} 39^{\prime} \mathrm{N}, 61^{\circ} 15^{\prime} \mathrm{E}\right), 14.04 .1993$, SVO; $20^{\top} \mathrm{O}^{\top}$ (AVG), Kopetdagh Mt. Range, Bäherden (c. $38^{\circ} 26^{\prime} \mathrm{N}, 57^{\circ}$ 26'E), 4.05.1993, SVO.

ADDITIONAL MATERIAL. Kyrgyzstan: $100^{7} \sigma^{7}, 4$ 우우 (AVG), North Kirgyzstan, Kirghizski Mt. Range, Ala-Archa River valley, Malinovka, c. $20 \mathrm{~km} \mathrm{~S}$ of Bishkek, 3.06.2004, SVO. TAлIKISTAN: $3 O^{7} O^{7}$ (ISEA; det. G.N. Azarkina), Khatlon Area, Dzhilantau Mt. Range, c. $2 \mathrm{~km} \mathrm{E}$ of Kul'bul'on kishlak $\left(37^{\circ} 53.970^{\prime} \mathrm{N}\right.$, $69^{\circ}$ $23.155^{\prime} \mathrm{E}$ ), dry stony scrubland vegetation with rocks, 970-1200 m a.s.1., 14.04.2019, AAF; 1 ○ (ISEA; det. G.N. Azarkina), same area, Gardaniushti Mt. Range, c. 2 km NW of Dakhanakiik kishlak $\left(38^{\circ} 12.883^{\prime} \mathrm{N}, 68^{\circ} 36.861^{\prime} \mathrm{E}\right)$, dry stony scrubland vegetation with rocks, $1130-1250 \mathrm{~m}$ a.s.1., 20.04.2019, AAF.

PREVIOUS RECORDS. Ashgabat, Pervomaiskoye, Magtymguly (=Kara-Kala), Kopetdagh Res., Firyuza (=Arçabyl), Badkhyz, Airi-Baba Mt., W Kopetdagh, Bäherden, Gaplan̆gyr Res., Sary-Kamysh Lake [Logunov et al., 1999], Zulfagarsky Mt. Range, Gezgyadyk Mt. Range [Logunov, Marusk, 2000a].

DISTRIBUTION. It is a European - Central Asian species, known from Iberian Peninsula in the west [Logunov, 2015] to Kyrgyzstan and Tajikistan in the east [Logunov et al., 1999], southward as far as Khorasan Province of Iran [Mirshamsi et al., 2013].

Pellenes (Pelmultus) nigrociliatus (Simon in L. Koch, 1875)

MATERIAL. TURKMENISTAN: 1 (ZMMU), SW Kopetdagh, Syunt-Khasardagh Res. (c. $\left.38^{\circ} 32^{\prime} \mathrm{N}, 56^{\circ} 25^{\prime} \mathrm{E}\right), 14.03 .1993$, EVP.

PREVIOUS RECORDS. Magtymguly (=Kara-Kala), Kalalegyoz Canyon [Logunov et al., 1999].

DISTRIBUTION. It is a widespread, south European Central Asian species, known eastward as far as Kyrgyzstan and Tajikistan [Logunov et al., 1999].

\section{Philaeus chrysops (Poda, 1761)}

MATERIAL. TURKMENISTAN: $50^{\top} \mathrm{O}^{\top}, 1+$ (ZMMU), Magtymguly (=Kara-Kala) Distr., Balkan Velayat, nr. Parkhai $\left(38^{\circ} 27^{\prime} 51^{\prime \prime} \mathrm{N}, 56^{\circ}\right.$ $14^{\prime} 58^{\prime \prime} \mathrm{E}$ ), foothills of Karaelchi Mt. Range, 2.05.1993, VLP \& EVP.

ADDITIONAL MATERIAL. UzBeKISTAN: $10^{7}$ (ZMMU; det. G.N. Azarkina), Novyi Margel, no date, Bankovsky. Pakistan: 19 (ZMMU), NWF, Hindukush, Chittal Distr., c. 7 km NE of Goboro-Bakh $\left(36^{\circ} 07^{\prime} \mathrm{N}, 71^{\circ} 23^{\prime} \mathrm{E}\right), 1.08 .2004$, SVO. TAJIKISTAN: $10^{\circ}$ (ISEA; det. G.N. Azarkina), right bank of Kafirnigan River, Romit kishlak $\left(38^{\circ} 43.226^{\prime} \mathrm{N}, 69^{\circ} 18.387^{\prime} \mathrm{E}\right)$, wet stony scrubland vegetation with rocks, $1200-1850 \mathrm{~m}$ a.s.1., 21-22.04.2019, AAF; $2 \mathrm{O}^{7} \mathrm{O}^{7}$ (ISEA; det. G.N. Azarkina), Khatlon Area, Gardaniushti Mt. Range, c. $2 \mathrm{~km}$ NW of Dakhanakiik kishlak $\left(38^{\circ} 12.883^{\prime} \mathrm{N}, 68^{\circ} 36.861^{\prime} \mathrm{E}\right)$, dry stony scrubland vegetation with rocks, $1130-1250 \mathrm{~m}$ a.s.l., 20.04.2019, AAF; $1 O^{7}, 1$ \& (ISEA; det. G.N. Azarkina), same area, Dzhilantau Mt. Range, c. $2 \mathrm{~km} \mathrm{E}$ of Kul'bul'on kishlak (37 $53.970^{\prime} \mathrm{N}, 69^{\circ} 23.155^{\prime} \mathrm{E}$ ), dry stony scrubland vegetation with rocks, 970-1200 m a.s.1., 14.04.2019, AAF; 1 + (ISEA; det. G.N. Azarkina), same area, Khodzhamumin Mt., c. 4 km SSE of Bose kishlak $\left(37^{\circ} 45.055^{\prime} \mathrm{N}, 69^{\circ} 38.709^{\prime} \mathrm{E}\right)$, wet stony scrubland vegetation with outcrops of saline rocks, 600-940 m a.s.1., 11.04.2019, AAF; 4 $\sigma^{7} \sigma^{7}, 5$ $\bigcirc \bigcirc$ (ISEA; det. G.N. Azarkina), same area, Shurobdariya River valley, Bal'dzhuvon kishlak $\left(8^{\circ} 18.835^{\prime \prime} \mathrm{N}, 69^{\circ} 41.011^{\prime \prime} \mathrm{E}\right)$, stony-clay slopes with meadow vegetation, 900-1000 m a.s.l., 15.04 2019, AAF; $1 \sigma^{7}$ (ISEA; det. G.N. Azarkina), same area, Tabaktsi Mt. Range, c. $3 \mathrm{~km}$ ENE of Kalininabad $\left(37^{\circ} 52.918^{\prime} \mathrm{N}, 68^{\circ}\right.$ $\left.58.719^{\prime} \mathrm{E}\right)$, dry stony scrubland vegetation, $1030-1280 \mathrm{~m}$ a.s.1., 18 04.2019, AAF. IrAN: 1 o (ISEA, 001.7974; det. G.N. Azarkina), Tehran, Elborz Mts (S slope), Tochal Mt. S slope at the higher aerial lift station $\left(35^{\circ} 51^{\prime} 31-35^{\prime \prime} \mathrm{N}, 51^{\circ} 23^{\prime} 58^{\prime \prime}-24^{\prime} 08^{\prime \prime} \mathrm{E}\right), 2939-2981$ m a.s.l., 2.06.2017, O.E. Kosterin.

PREVIOUS RECORDS. Ashgabad, Bolshoy Balkhan Mts [Wesołowska, 1996], Koytendag Res. [Stoev et al., 2019].

DISTRIBUTION. A trans-Palaearctic subboreal-subtropical species [Logunov, Marusik, 2000b]. 1872)

Plexippoides flavescens (O. Pickard-Cambridge,

MATERIAL. Turkmenistan: 1 (ZMMU), Magtymguly (=Kara-Kala) Distr., Balkan Velayat, nr. Parkhai $\left(38^{\circ} 27^{\prime} 51^{\prime \prime} \mathrm{N}, 56^{\circ}\right.$ $14^{\prime} 58^{\prime \prime} \mathrm{E}$ ), hills of Palaeogene clays, 15.12.1992, VLP; 1 (ZMMU), same distr. and velayat, Karaelchi Mt. Range $\left(38^{\circ} 30^{\prime} \mathrm{N}, 56^{\circ} 15^{\prime} \mathrm{E}\right)$, $400 \mathrm{~m}$ a.s.1., 17.12.1992, VLP; $3 \mathrm{O}^{7} \mathrm{O}^{7}$ (ZMMU), same distr. and velayat, S slope of Karaelchi Mt. Range $\left(38^{\circ} 30^{\prime} \mathrm{N}, 56^{\circ} 15^{\prime} \mathrm{E}\right), 600 \mathrm{~m}$ a.s.1., 18.12.1992, VLP; 1 ○', 2 क् (ZMMU), SW Kopetdagh, Syunt-Khasardagh Res. (c. $\left.38^{\circ} 32^{\prime} \mathrm{N}, 56^{\circ} 25^{\prime} \mathrm{E}\right), 7-14.03 .1993$, EVP,

PREVIOUS RECORDS. Gezgyadyk Mt. Range [Wesołowska, 1996], Magtymguly (=Kara-Kala), Akhalsky Velayat, Kyzyl-Dzhar, Zulfagarsky Mt. Range, Gezgyadyk Mt. Range, Bagly-Dara, Kumasan, Chardzhou, Kenderly [Logunov, Rakov, 1998].

DISTRIBUTION. An eastern Mediterranean - Central Asian subboreal species, known from the Arabian Peninsula and Sinai [Wesołowska, van Harten 2007, 2011], throughout Iran and Turkmenistan [Logunov, Rakov, 1998; Mirshamsi et al., 2013], to Afghanistan and Pakistan in the east [Logunov, Zamanpoore, 2005; Bauer at al., 2015].

\section{Pseudicius courtauldi Bristowe, 1935}

MATERIAL. TURKMENistan: $20^{7} \sigma^{\top}$ (ZMMU), Amudariya Res. (c. $39^{\circ} 39^{\prime} \mathrm{N}, 62^{\circ} 51^{\prime} \mathrm{E}$ ), Kabakly site, tugai, 19.04 .1993 , VLP.

ADDITIONAL MATERIAL. UZBEKISTAN: $3 \sigma^{7} \sigma^{7}$ (AVG), Babatagh Mt. Range, Ak-Mechet, 26.04.1994, SVO.

PREVIOUS RECORDS. Amudariya Res. [Wesołowska, 1996], Kara-Belent Mt. [Logunov, Rakov, 1998].

DISTRIBUTION. The species is known from Minor Asia in the west, throughout Central Asia to NW China in the east [Logunov, 1993; Wesołowska, 1996; Logunov, Rakov, 1998; present data].

\section{Pseudomogrus albocinctus (Kroneberg, 1875)}

MATERIAL. TURKMENISTAN: 1 (ZMMU), Amudariya Res. (c. $39^{\circ} 39^{\prime} \mathrm{N}, 62^{\circ} 51^{\prime} \mathrm{E}$ ), Kabakly site, tugai, 19.04.1993, VLP.

PREVIOUS RECORDS. Deinau, Sultanbent, Amudariya Res. [Logunov, Marusik, 2003: sub Yllenus a.].

DISTRIBUTION. The species is known from Armenia/ Turkey in the west to W Mongolia in the east [Logunov, Marusik, 2003: Map 8; sub Yllenus a.].

Pseudomogrus pseudovalidus (Logunov et Marusik, 2003)

Figs $37-42$.

MATERIAL. TURKMENISTAN: 1 + (ZMMU), Lebap Velayat, nr. Seidi $\left(39^{\circ} 29^{\prime} \mathrm{N}, 62^{\circ} 54^{\prime} \mathrm{E}\right)$, sand-clay desert, 12.04 .1993 , VLP.

PREVIOUS RECORDS. Kizyl-Su, Karadagh Mts [Logunov, Marusik, 2003: sub Yllenus p.].

DISTRIBUTION. Central Asian (Turanian) endemic species, known from a few localities in Kazakhstan and Turkmenistan only [Logunov, Marusik, 2003: Map 19; sub Yllenus p.; Ponomarev, Dvadnenko, 2012: sub Yllenus p.]. 


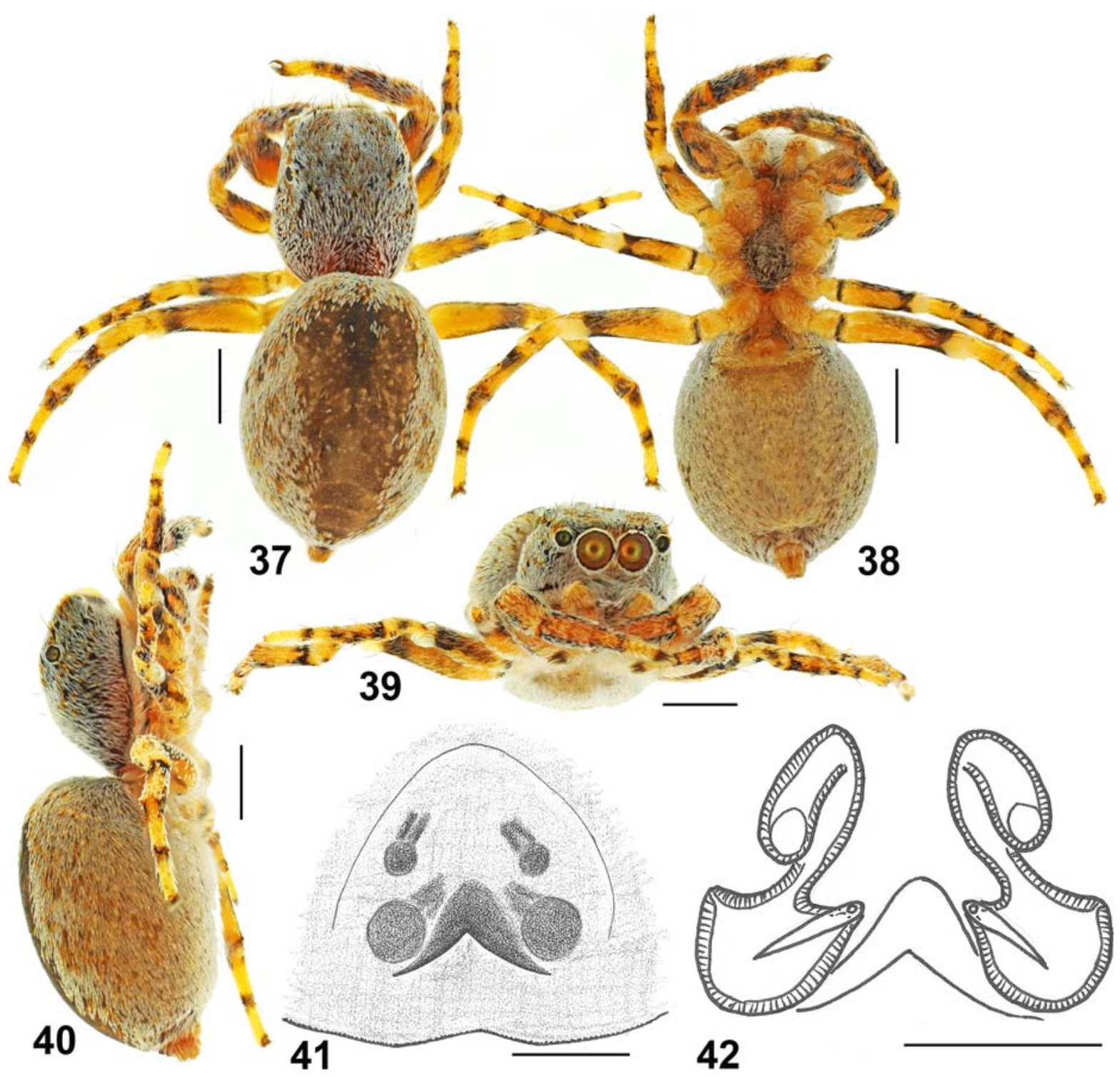

Figs 37-42. Female of Pseudomogrus pseudovalidus (Logunov et Marusik, 2003) from Seidi, Turkmenistan: 37 — general appearance, dorsal view; 38 - ditto, ventral view; 39 - ditto, frontal view; 40 - ditto, lateral view; 41 - epigyne, ventral view; $42-$ spermathecae, dorsal view. Scale bars: $(41,42) 0.1 \mathrm{~mm},(37-40) 1 \mathrm{~mm}$.

Рис. 37-42. Самка Pseudomogrus pseudovalidus (Logunov et Marusik, 2003) из Сейди, Туркменистан: 37 - общий вид, сверху; 38 - тоже, снизу; 39 - тоже, спереди; 40 - тоже, сбоку; 41 - эпигина, вид снизу; 42 - сперматека, вид сверху. Масштаб: (41, 42) 0,1 мм, (37-40) 1 мм.

COMMENTS. Here we have presented colour photographs of the female's general appearance of this species (Figs 37-40) for the first time. The body colour and colour pattern of the studied female correspond and are complementary to the detailed description provided by Logunov \& Marusik [2003: 61], except for the absence of reddish clypeal hairs, which are available in the paratype female.

Rudakius spasskyi (Andreeva, Hęciak et Prószyński, 1984)

MATERIAL. TURKMENISTAN: 1 (ZMMU), Amudariya Res. (c. $39^{\circ} 39^{\prime} \mathrm{N}, 62^{\circ} 51^{\prime} \mathrm{E}$ ), Kabakly site, c. $11 \mathrm{~km}$ S of Kabakly, nr.
Uchkersen kishlak, tugai, on Populus diversifolia, 16.04.1993, VLP; $1 O^{7}$ (AVG), SW Kopetdagh, nr. Magtymguly (=Kara-Kala), 29.03.1993, SVO.

PREVIOUS RECORDS. Amudariya Res., Farab, SyuntKhasardagskii Res. [Wesołowska, 1996: sub. Pseudicius s.], Magtymguly (=Kara-Kala), Aidere, Kampyr-Tepa, Ashgabad, Kysyl-Su, Repetek, Turkmenbashi (=Krasnovodsk) [Logunov, Rakov, 1998: sub. Pseudicius s.].

DISTRIBUTION. A Central Asian (Turanian) species, known from Azerbaijan to Tajikistan [Wesołowska, 1996; Logunov, Rakov, 1998; Logunov, Guseinov, 2002; all sub. Pseudicius s.; present data], southward as far as northern Iran [Logunov et al., 2007: sub. Pseudicius s.]. 


\section{Salticus tricinctus (C.L. Koch, 1846)}

MATERIAL. TURKMENISTAN: $3 \sigma^{7} \sigma^{\top}$ (ZMMU), Charshanga Distr., Lebap Velayat, c. $3 \mathrm{~km} \mathrm{E}$ of Bazar-Depe kishlak (c. $37^{\circ} 48^{\prime} \mathrm{N}$, $66^{\circ} 30^{\prime} \mathrm{E}$ ), Kugitangh Res., low-mountain relief, 31.03.1993, VLP; $2 \mathrm{O}^{\top} \mathrm{O}^{7}$ (ZMMU), Amudariya Res. (c. $\left.39^{\circ} 39^{\prime} \mathrm{N}, 62^{\circ} 51^{\prime} \mathrm{E}\right)$, Kabakly site, tugai, 19.04.1993, VLP

ADDITIONAL MATERIAL. KyrgyzSTAN: 19 (AVG), Sandalashski Mt. Range, Kuru-Terek, 10.07.1998, SVO. Iran: 19 (ISEA, 001.7977; det. G.N. Azarkina) Markazi Ostan, Shazand

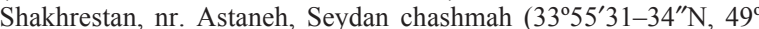
20'53-57"E), 2277-2287 m a.s.1., 20.05.2017, O.E. Kosterin.

PREVIOUS RECORDS. Amudariya Res. [Wesołowska, 1996], Magtymguly (=Kara-Kala), Turkmenbashi (=Krasnovodsk), Morgunovka [Logunov, Rakov, 1998].

DISTRIBUTION. An eastern Mediteranean - Central Asian species, known from Turkey, throughout the Caucasus and Central Asia to Afghanistan [Ponomarev, 2005; Logunov, 2015; Ponomarev et al., 2017].

\section{Thyene imperialis (F.W. Rossi, 1846)}

MATERIAL. TURKMENISTAN: $10^{\top}$ (ZMMU), SW Kopetdagh, Syunt-Khasardagh Res. (c. $\left.38^{\circ} 32^{\prime} \mathrm{N}, 56^{\circ} 25^{\prime} \mathrm{E}\right), 14.03 .1993$, EVP.

ADDITIONAL MATERIAL. TAJIKISTAN: 1 o (ZMMU; det. G.N. Azarkina), 'Kurgan-Tyubinskii', sparrow nest, 5.11.1946, coll.?. Pakistan: 1 (MMUE), Lahore, canal bank, 21.09.2005, A Butt; $1 O^{7}$ (MMUE), Lahore, rice field, 21.06.2004, A. Butt; $10^{7}, 1$ + (MMUE), NWF, Peshawar, Forestry Campus of Agricultural University, 14-26.08.2004, SVO; 1 o', 1 ㅇ (MMUE), Karachi, 13.07.2013, M. Kazim.

PREVIOUS RECORDS. SW Kopetdagh (Magtymguly) [Wesołowska, 1996]; Turkmenbashi (=Krasnovodsk), Kuhitangtau Mt. Range, Sharlouk, Aidere, Sovkhoz Karakumsky [Logunov, Rakov, 1998].

DISTRIBUTION. A trans-Eurasian subboreal-subtropical species, known from the Canaries, throughout the Mediterranean and northern Africa, to China and India [Logunov, 2015].

\section{Yllenus turkestanicus Logunov et Marusik, 2003}

MATERIAL. TURKMENISTAN: 1 (ZMMU), $70-80 \mathrm{~km} \mathrm{~N}$ of Turkmenbashi (=Krasnovodsk), Chul'-Mamed'Kum sands $\left(40^{\circ}\right.$ $35^{\prime} \mathrm{N}, 53^{\circ} 05^{\prime} \mathrm{E}$ ), nr. Adzhikuyu, 25-30.05.1985, EAK.

PREVIOUS RECORDS. Chul'-Mamed'Kum sands, Amudariya Res., Kankakyr Height, Charyshly well, Shakhsenem well, Repetek, Kizyl-Su, Ashgabat, W Kopetdagh, Lake Sarykamysh, Takhta Distr. [Wesołowska, 1996: sub. Y. flavociliatus; Logunov, Marusik, 2003].

DISTRIBUTION. Central Asian (Turanian) endemic; restricted western and southern Kazakhstan, Uzbekistan and Turkmenistan [Logunov, Marusik, 2003: Map 23; Ponomarev, 2008].

\section{Discussion}

Based on earlier and present findings, the salticid fauna of Turkmenistan currently includes 99 species, which is less than those of the larger neighbouring territories of Kazakhstan (156 species) [Mikhailov, 2016; Metzner, 2019] and Iran (108 species) [Zamani et al., 2019], and a bit more that those of smaller Azerbaijan (88 species), Uzbekistan (64), Tajikistan (64) and Kyrgyzstan (87) [Mikhailov, 2016]. Metzner [2019] listed 109 salticid species as occurring in Turk- menistan, but his list uncritically includes a number of incorrect records, for instance, of such species as $\mathrm{He}$ liophanus melinus Simon, 1889, Macaroeris nidicolens (Walckenaer, 1802), Pellenes limbatus Kulczyński, 1895, P. simoni (O. Pickard-Cambridge, 1872), $P$. tripunctatus (Walckenaer, 1802), Sitticus terebratus (Clerck, 1758), Yllenus flavociliatus Simon, 1895, etc. For example, as shown by Wesołowska [1996], the records of European $H$. melinus from Turkmenistan were to be referred to Heliophanus patagiatus Thorell, 1875. The distribution of $M$. nidicolens is restricted in its eastward limit by Turkey [Logunov, 2015]. All the records of $P$. tripunctatus from Middle Asia, including Turkmenistan, were mistaken and should actually be referred to Pellenes seriatus (Thorell, 1875) [Logunov, Marusik, 1994]. Equally, the records of $P$. limbatus are to be referred to Pellenes turkmenicus Logunov, Marusik et Rakov, 1999, whereas those of P. simoni to Pellenes geniculatus (Simon, 1868) (see Logunov et al. [1999]). The records of Y. flavociliatus from Turkmenistan were mistaken and actually refer to those of Yllenus turkestanicus (see Logunov \& Marusik [2003]); and so on.

The current state of knowledge of Salticidae could be considered satisfactory but not complete. For instance, each of the salticid faunas of equally diverse neighbouring faunas of Iran and Kazakhstan contain 40 genera [Metzner, 2019; Zamani et al., 2019], compared to 33 salticid genera found in Turkmenistan [Metzner, 2019; present data]. The following seven genera, which are known from the neighbouring countries, are likely to be found in Turkmenistan as well: Chinattus Logunov, 1999 (1 species), Habrocestum Simon, 1876 (1-2 species), Macaroeris Wunderlich, 1992 (1 species), Mexcala Peckham et Peckham, 1902 (1 species), Pseudeuophrys Dahl, 1912 (1-2 species), Sibianor Logunov, 2001 (1-2 species), and Talavera Peckham et Peckham, 1909 (1-2 species). Yet, more species records and/or descriptions are to be expected in the following five genera: Langona Simon, 1901, Menemerus Simon, 1868, Pellenes Simon, 1876, Proszynskiana Logunov, 1996, Salticus Latreille, 1804, and Yllenus (s. lat.) Simon, 1868. A rather conservative estimate of the real diversity of Turkmen Salticidae should be around 120-130 species.

Acknowledgements. We want to thank the following colleagues who made their salticid collections available for this study: Edouard A. Khachikov (Rostov-on-Don, Russia), Vladimir L. Perepechaenko (Belorechensk, Russia), the late Sergei V. Ovtchinnikov (Bishkek, Kyrgyzstan), and Elena V. Prokopenko (Donetsk, Ukraine). We also wish to sincerely thank Galina N. Azarkina (Novosibirsk, Russia) for the identification of our Aelurillus samples, providing us with additional salticid records from Middle Asia and making the maps 2 and 3. We are most grateful to Vladimir Yu. Shmatko (Rostov-on-Don, Russia) for making digital photos of three species (Figs $8-11$ and 20-25) and to Tony Hunter (Liverpool, UK) for allowing one of us (DL) to use the digital facilities at the World Museums of Liverpool (UK). Finally, we want to thank Viktor Ya. Fet (Huntington, 
USA) for his kind help with finding correct coordinates for the localities from SW Kopetdagh.

\section{References}

Azarkina G.N. 2003. Aelurillus ater (Kroneberg, 1875) and related species of jumping spiders in the fauna of middle Asia and the Caucasus (Aranei: Salticidae) // Arthropoda Selecta. Vol.11. No.1. P.89-107.

Azarkina G.N., Mirshamsi O. 2014. Description of a new Aelurillus species from Khorasan province of Iran, with comments on A. concolor Kulczyñski, 1901 (Araneae: Salticidae) // Zoology in the Middle East. Vol.60. No.1. P.82-91. doi:10.1080/ 09397140.2014 .892353

Azarkina G.N., Zamani A. 2019. The Aelurillina Simon, 1901 (Aranei: Salticidae) of Iran: a check-list and three new species of Aelurillus Simon, 1884 and Proszynskiana Logunov, 1996 // Arthropoda Selecta. Vol.28. No.1. P.83-97.

Bauer T., Freudenschuss M., Grabolle A. 2015. Plexippoides flavescens (O. Pickard-Cambridge, 1872) and Menemerus marginatus (Kroneberg, 1875), new records for Pakistan (Aranei: Salticidae) // Arthropoda Selecta. Vol.24. No.1. P.87-90.

Blick T., Marusik Yu.M. 2018. Three junior synonyms of jumping spider genera (Araneae: Salticidae) // Arthropoda Selecta. Vol.27. No.3. P.237-238.

Kropf C., Blick T., Brescovit A.D., Chatzaki M., Dupérré N., Gloor D., Haddad C.R., Harvey M.S., Jäger P., Marusik Yu.M., Ono H., Rheims C.A., Nentwig W. 2019. How not to delimit taxa: a critique on a recently proposed "pragmatic classification" of jumping spiders (Arthropoda: Arachnida: Araneae: Salticidae) // Zootaxa. Vol.4545. No.3. P.444-446. doi:10.11646/zootaxa.4545.3.10

Logunov D.V. 1993. Notes on two salticid collections from China (Araneae Salticidae) // Arthropoda Selecta. Vol.2. No.1. P.4959.

Logunov D.V. 1995a. New and little known species of the jumping spiders from central Asia (Araneae: Salticidae) // Zoosystematica Rossica. Vol.3. P.237-246.

Logunov D.V. 1995b. The genus Mogrus (Araneae: Salticidae) of central Asia // European Journal of Entomology. Vol.92. P.589604

Logunov D.V. 1996. Salticidae of Middle Asia. 3. A new genus, Proszynskiana gen.n., in the subfamily Aelurillinae (Araneae, Salticidae) // Bulletin of the British arachnological Society. Vol.10. No.5. P.171-177.

Logunov D.V. 2010. Taxonomic notes on a collection of jumping spiders from Iran (Araneae, Salticidae) // Bulletin of the British arachnological Society. Vol.15. No.3. P.85-90.

Logunov D.V. 2015. Taxonomic-faunistic notes on the jumping spiders of the Mediterranean (Aranei: Salticidae) // Arthropoda Selecta. Vol.24. No.1. P.33-85.

Logunov D.V., Marusik Yu.M. 1994. New data on the jumping spiders of the Palearctic fauna (Aranei Salticidae) // Arthropoda Selecta. Vol.3. No.1-2. P.101-115.

Logunov D.V., Marusik Yu.M. 1999. A brief review of the genus Chalcoscirtus Bertkau, 1880 in the faunas of Central Asia and the Caucasus (Aranei: Salticidae) // Arthropoda Selecta. Vol.7. No.3. P.205-226.

Logunov D.V., Marusik Yu.M. 2000a (for 1999). Miscellaneous notes on Palaearctic Salticidae (Arachnida: Aranei) // Arthropoda Selecta. Vol.8. No.4. P.263-292.

Logunov D.V., Marusik Yu.M. 2000b. Catalogue of the jumping spiders of northern Asia (Arachnida, Araneae, Salticidae). Moscow: KMK Scientific Press, 299 pp.

Logunov D.V., Marusik Yu.M. 2003. A revision of the genus Yllenus Simon, 1868 (Arachnida, Araneae, Salticidae). Moscow: KMK Scientific Press Ltd. 167pp.

Logunov D.V., Marusik Yu.M., Mozaffarian F. 2002. Faunistic review of the jumping spiders of Iran (Aranei: Salticidae) // Arthropoda Selecta. Vol.10. No.2. P.155-167.

Logunov D.V., Marusik Yu.M., Rakov S.Yu. 1999. A review of the genus Pellenes in the fauna of Central Asia and the Caucasus
(Araneae, Salticidae) // Journal of Natural History. Vol.33. P.89-148.

Logunov D.V., Mirshamsi O., Musavi S., Shayestehfar A. 2013. New faunistic records of Salticidae (Aranei) from Khorasan Province of Iran // Arthropoda Selecta. Vol.22. No.4. P.379381.

Logunov D.V., Rakov S.Yu. 1998. Miscellaneous notes on Middle Asian jumping spiders (Aranei: Salticidae) // Arthropoda Selecta. Vol.7. No.2. P.117-114.

Logunov D.V., Vazirianzadeh B., Moravvej S.A., Navidpour S. 2007 (for 2006). New faunistic records of the jumping and crab spiders (Aranei: Salticidae, Thomisidae and Philodromidae) from Iran // Arthropoda Selecta. Vol.15. No.3. P.225228.

Logunov D.V., Zamanpoore M. 2005. Salticidae (Araneae) of Afghanistan: an annotated check-list, with descriptions of four new species and three new synonyms // Bulletin of the British arachnological Society. Vol.13. Pt.6. P.217-232.

Metzner H. 2019. Jumping spiders (Arachnida: Araneae: Salticidae) of the world, online at: https://www.jumping-spiders.com (accessed on 1st Desember 2019).

Mikhailov K.G. 2016. Advances in the study of spider fauna (Aranei) of Russia and adjacent regions: a 2015 update // Vestnik Zoologii. Vol.50. No.4. P.309-320. DOI 10.1515/vzoo-20160038

Mikhailov K.G., Fet V.Ya. 1994. Fauna and zoogeography of spiders (Aranei) of Turkmenistan // Fet V., Atamuradov K.I. (eds.). Biogeography and ecology of Turkmenistan, Kluwer Acad. Publ. P.499-524.

Mirshamsi O., Shayestehfar A., Musavi S., Hamta A. 2013. New data on the jumping spiders from northeast of Iran (Aranei: Salticidae) // Iranian Journal of Animal Biosystematics. Vol.9. No.2. P.117-123.

Namaghi H.S., Safari A., Entezari E., Zamani A. 2016. New faunistic records of spiders (Araneae) from northeastern Iran // Zoology and Ecology. Vol.26. P.18-21.

Ono H. 1988. A revisional study of the spider family Thomisidae (Arachnida, Araneae) of Japan. Tokyo: National Science Museum. 252 pp.

Ponomarev A.V. 2008. [The additional data to the spider fauna (Aranei) of the south-east of Russian plain] // Vestnik Yuzhnogo Nauchnogo Tsentra Rossijskaja Akademija Nauk, Rostov. Vol.4. P.78-86 [in Russian].

Ponomarev A.V. 2005. [New and interesting findings of spiders (Aranei) from the southeast part of Europe] // Vestnik Yuzhnogo Nauchnogo Tsentra Rossijskaja Akademija Nauk, Rostov. Vol.1. No.4. P.78-86 [in Russian].

Ponomarev A.V., Abdurakhmanov G.M. 2014. [Spiders (Aranei) of the shore and islands of the northern part of Caspian Sea] // Yugh Rossii: ekologiya, razvitie. No.1. P. 76-121 [in Russian]. DOI: 10.18470/1992-1098-2014-1-76-121

Ponomarev A.V., Alekseev S.K., Kozminykh V.O., Shmatko V.Yu. 2017. Spiders (Arachnida: Aranei) of Stavropol Province, Russia // Arthropoda Selecta. Vol.26. No.2. P.155-173.

Ponomarev A.V., Dvadnenko K.V. 2012. [Notes on the taxonomy and fauna of spiders (Aranei) from the south of Russia and western Kazakhstan] // Yugh Rossii: ekologiya, razvitie. No.4. P.42-53 [in Russian].

Ponomarev A.V., Khnykin A.S. 2013. [Spiders (Aranei) of Volgograd and its vicinities] // Yugh Rossii: ekologiya, razvitie. No.4. P.109-136 [in Russian]. DOI: 10.18470/1992-10982013-4-109-136

Ponomarev A.V., Prishutova Z.G. 2017. [Terrestrial spiders (Aranei) of Vodnyi Island (Manych-Gudilo Lake)] // Nauka Yuga Rossii. Vol.13. No.2. P.60-65 [in Russian].

Ponomarev A.V., Shokhin I.V., Terskov E.N., Shmatko V.Yu. 2018. [Preliminary data on the spider fauna (Aranei) of Taman Peninsula and Tuzla Island (Russia)] // Caucasian Entomological Bulletin. Vol.14. No.2. P.121-129 [in Russian].

Prószyński J. 1979. Systematic studies on East Palearctic Salticidae III. Remarks on Salticidae of the USSR // Annales Zoologici, Warszawa. Vol.34. P.299-369. 
Prószyński J. 1989. Salticidae (Araneae) of Saudi Arabia // Fauna Saudi Arabia. Vol.10. P.31-64.

Prószyński J. 2018. Review of genera Evarcha and Nigorella, with comments on Emertonius, Padilothorax [sic], Stagetillus, and description of five new genera and two new species (Araneae: Salticidae) // Ecologica Montenegrina. Vol.16. P.130-179.

Rakov S.Yu. 1997. A review of the spider genus Evarcha Simon, 1902 in Middle Asia (Aranei Salticidae) // Arthropoda Selecta. Vol.6. No.1/2. P.105-112.

Rakov S.Yu., Logunov D.V. 1997a. A critical review of the genus Heliophanus C. L. Koch, 1833, of Middle Asia and the Caucasus (Aranei, Salticidae) // Arthropoda Selecta. Vol.5 (for 1996). No.3/4. P.67-104.

Rakov S.Yu., Logunov D.V. 1997b. Taxonomic notes on the genus Menemerus Simon, 1868 in the fauna of Middle Asia (Araneae, Salticidae) // Proceedings of the 16th European Colloquium of Arachnology. P.271-279.

Stoev P., Deltshev C., Marusik Yu.M., Fet V., Kovařík F. Guéorguiev B., Dedov I., Ljubomirov T., Chobanov D., Menliev S. 2019. Surface-dwelling invertebrates // Welch G., Stoev P. (eds.). A report of RSPB-supported scientific research at
Koytendag State Nature Reserve, East Turkmenistan. Sofia: Pensoft Publishers. P.31-36.

Wesołowska W. 1996. New data on the jumping spiders of Turkmenistan (Aranei, Salticidae) // Arthropoda Selecta. Vol.5. No.1-2. P.17-53

Wesołowska W., van Harten A. 2007. Additions to the knowledge of jumping spiders (Araneae: Salticidae) of Yemen // Fauna of Arabia. Vol.23. P.189-269.

Wesołowska W., van Harten A. 2011. Order Araneae, family Salticidae: Additions and the descriptions of a new species // van Harten A. (ed.). Arthropod Fauna of the UAE. Dar Al Ummah, Abu Dhabi 4. P.23-28.

WSC 2019. World Spider Catalog. Version 20.5. Natural History Museum Bern, online at: http://wsc.nmbe.ch, accessed on 14 December 2019. doi: 10.24436/2

Zamani A., Mirshamsi O., Marusik Yu.M., Moradmand M. 2019. The Checklist of the Spiders of Iran. Version 2020; online at: www.spiders.ir (accessed on 1st Dec 2019).

Responsible editor K.G. Mikhailov 